\title{
L'urbanisation des zones côtières: utilisation des sols, implications morphologiques et environnementales. Le cas de la ville de Jeddah
}

\author{
Dr. Abdoul Jelil NIANG ${ }^{1 *} \&$ Dr. Ibrahim ASCOURA ${ }^{1,2 * *}$ \\ ${ }^{1}$ Department of Geography, College of Social Sciences, Umm Al-Qura University Makkah \\ Saudi Arabia \\ ${ }^{2}$ Department of Geography, College of Arts, Helwan University, Cairo, Egypt. \\ Email :* anniang@uqu.edu.sa - ajeniang@yahoo.fr, ** ieascoura@uqu.edu.sa,
}

ascoura@hotmail.com

\section{Résumé}

Jeddah est la plus grande ville côtière du royaume et abrite le premier port de la mer Rouge, représentant la porte d'entrée des pèlerins pour rejoindre la Mecque. Cette cité historique, coincée entre la mer Rouge à l'ouest et la zone montagneuse du Hedjaz à l'est, est la deuxième ville d'Arabie Saoudite en termes d'extension spatiale et de population, après la capitale Riyad. Elle a connu une croissance urbaine sans précèdent, lors des décennies 70 et 80 , qui s'est traduite par une littoralisation intensive. Cette étude se focalise sur cette pression anthropique continue sur la zone côtière .Une approche de suivi spatiotemporel de l'extension urbaine sur la mer, par l'utilisation des Systèmes d'information géographique (SIG) et des données de télédétection multisources a été adoptée. Les superficies des remblaiements successifs ont été cartographiées pour différentes périodes de 1965 à 2020. Les diverses affectations dont elles ont fait l'objet sont analysées, ainsi que changements morphologiques et environnementaux qu'elles entrainent. Les résultats montrent que la surface totale des terrepleins sur la période d'observation atteint environ $33 \mathrm{~km}^{2}\left(0,59 \mathrm{~km}^{2} / \mathrm{an}\right.$ en moyenne) dont 24,5 $\mathrm{km}^{2}$ entre 1972 et 1986, période de boom économique où Jeddah a enregistré sa plus grande expansion urbaine. Lors des diverses phases de la période d'étude, le port de Jeddah a gagné environ $11,8 \mathrm{~km}^{2}$ de superficie sur la mer, soit $94,4 \%$ de la zone industrialo-portuaire actuelle. Ainsi ce sont des zones maritimes, des récifs coraliens et d'ilots qui ont été transformées en terrains urbanisés dont les utilisations sont variées; installations portuaires $(36,1 \%)$, gouvernementale (21,5\%), service Public (16.4), récréative/touristique (15,2\%), ou résidentielle et commerciale $(10,8 \%)$. Ces mutations impliquent à leur tour un bouleversement de l'environnement marin côtier; création de lagunes semi-fermées et d'ilots artificiels, disparition de récifs coralliens, d'ilots et une modification de la morphologie de cette zone côtière.

Mots clefs : urbanisation, zone côtière, terre-plein, utilisation des sols, modifications environnementales, SIG, télédétection, Jeddah, Arabie Saoudite 
تحضر الحيز الساحلي: استخدام الأراضي والآثار المورفولوجية والبيئية

$$
\begin{aligned}
& \text { حالة مدينة جدة } \\
& \text { د. عبد الجليل انيانلك1، د. إبر اهيم السبد عسكورة1،2 } \\
& 1 \text { قسم الجغر افيا، كلية العلوم الاجتماعية، جامعة أم القرى، المملكة العربية السعودية } \\
& 2 \text { قسم الجغر افيا، كلبة الآداب، جامعة حلو ان، مصر }
\end{aligned}
$$

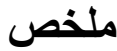

مدينة جدة هي أكبر الددن الساحلية بالمملكة العربية السعودية، وهي موطن أول ميناء على البحر

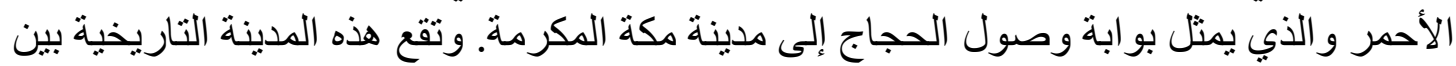

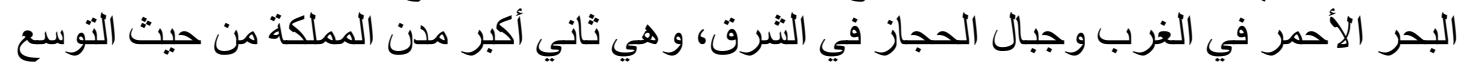

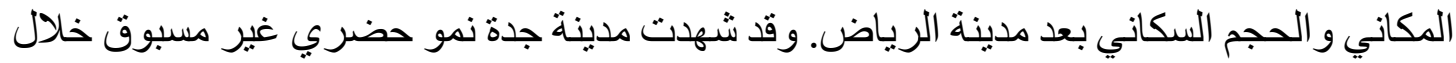

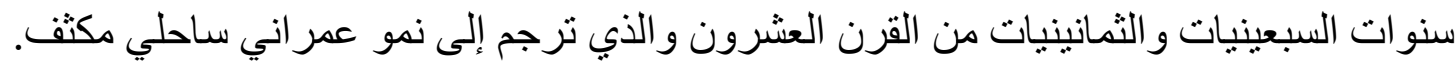

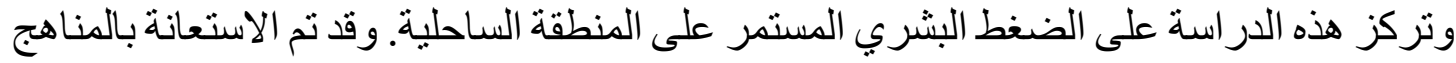

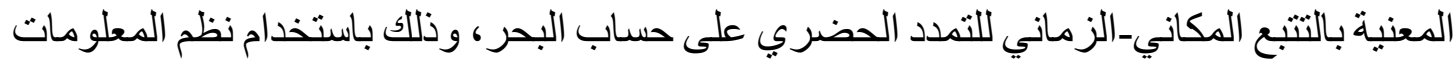

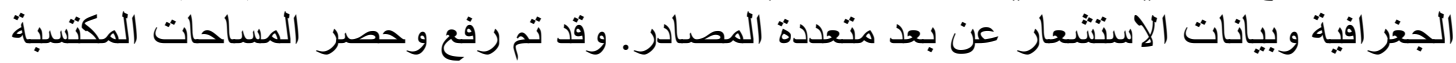

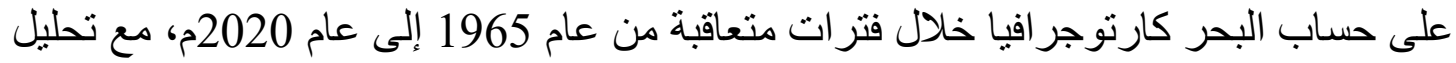

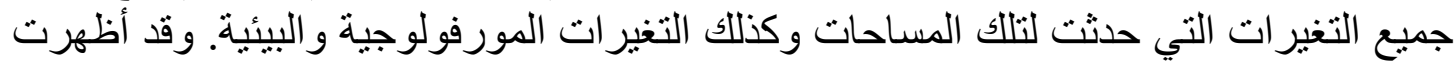

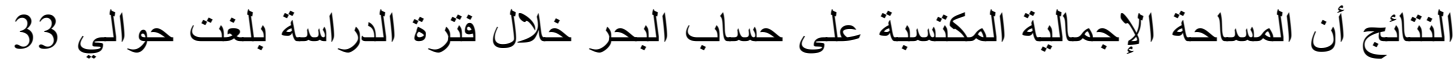

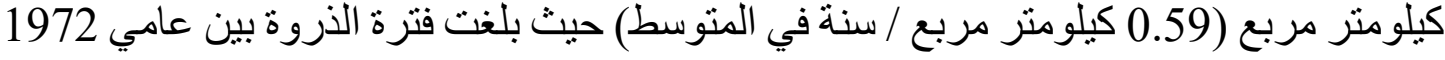

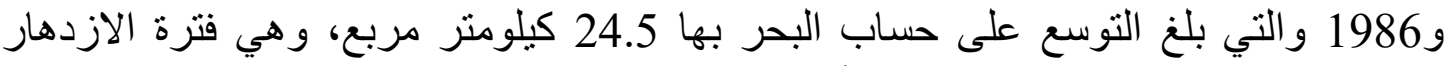

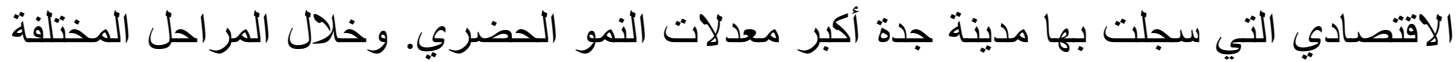

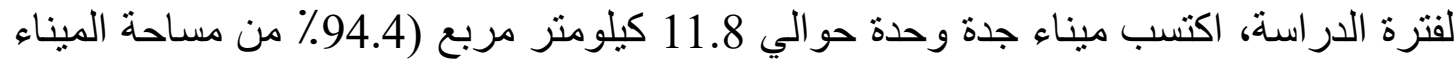

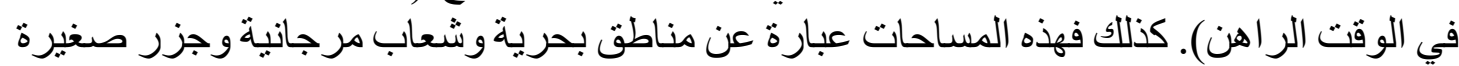

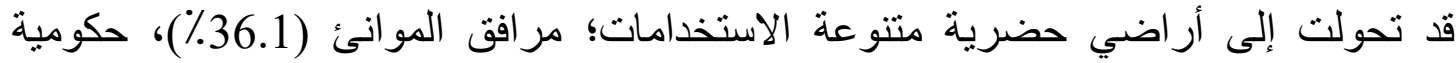

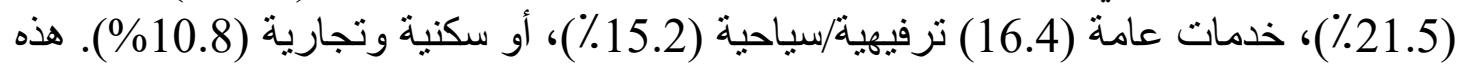

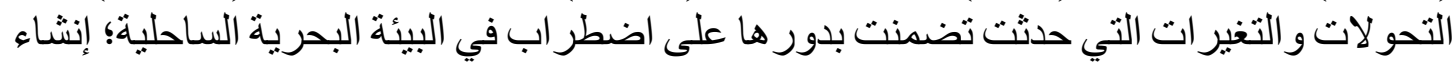

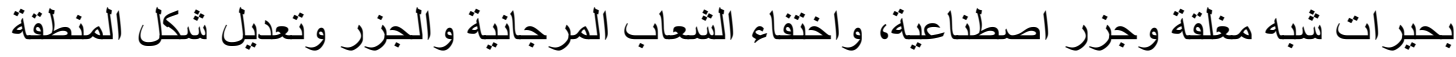
الساحلية.

الكلمات المفتاحية: التحضر - الحيز الساحلي_ استخدام الأراضيـ الآثار المورفولوجية والبيئية ـ

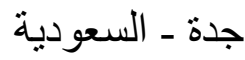




\section{1- Introduction}

Les zones littorales, qui sont des interfaces dynamiques, sont actuellement soumises, à travers le monde, à d'importantes pressions anthropiques, liées notamment à la croissance urbaine sans précédent qui s'y développe (Tezuka Akira et Takahashi Nobuo 1998 ; Le Berre et Robert 2017). L'extension spatiale des villes côtières et l'occupation progressive du littoral, se fait souvent au détriment de la mer. Il en résulte une croissance des surfaces urbaines artificialisées que l'homme, grâce aux progrès technologiques, est parvenu à aménager et à élargir pour l'implantation d'infrastructures et d'équipements, de zones résidentielles, industrielles ou touristiques, avec tous les risques et conséquences induits (El-Raey, 2009, Al-Sheikh, 2011, Basaham et al, 2006, Karim et al.,2018, Martínez et al., 2007 ; Mansour et Madkour. 2015 ; Le Berre 2017). Selon EEA (2006), la croissance des surfaces urbaines artificialisées le long des côtes européennes entre 1990 et 2000 a atteint $10 \%$ sur la côte méditerranéenne et $15 \%$ sur la côte atlantique. L'accroissement de la surface de ces terre-pleins côtiers et le changement constant de la morphologie des côtes, a fait de l'homme un agent géomorphologique majeur des littoraux, selon Cooper et Alonso (2006). Dans ce contexte on observe également un changement significatif et une grande diversité au niveau de l'utilisation des sols dans ces nouvelles terres gagnées sur la mer.

Les différentes études menées sur la ville de Jeddah dans le domaine de l'extension urbaine se sont concentrées essentiellement sur les vecteurs $\mathrm{du}$ développement urbain (Abdu et al. 2002), la planification urbaine, les tendances spatiales et les directions de l'étalement urbain (Abdulghani, 1983 ; Helmi 2015, Al Enezi, 2019) ou sur les conséquences environnementales de l'urbanisation littorale (Bahasam et al. 2006, Mansour et Madkour, 2015). D'autres auteurs se sont intéressés à l'occupation des sols de manière générale, dans le sillage de l'extension urbaine (Abdulghani, 1983, Al Enezi, 2019). Aljoufie (2020) a étudié les interactions entre l'utilisation des sols (les centres commerciaux notamment), le transport public et le développement durable. L'urbanisation dans la zone côtière et ses conséquences environnementales n'ont fait l'objet que de rares études tandis que l'évolution spatiotemporelle des terre-pleins n'a pas été étudiée, à notre connaissance. Basaham et al., (2006) ont abordé les conséquences de l'urbanisation incontrôlée dans le secteur de Sharm Abhur. Aboulela et al. (2020) ont analysé les changements survenus à certains 
endroits du littoral de Jeddah de 1972 à 2016 et les tendances futures de l'évolution de ces zones. D'où l'intérêt d'aborder la question de la cartographie des surfaces acquises au détriment de la mer le long du littoral de Jeddah ainsi que leur utilisation et l'analyse de l'implication morphologique et environnementale que cela entraine.

L'importance de la ville de Jeddah porte l'empreinte de nombreuses causes : historiques, démographiques, économiques et urbaines. Depuis des siècles, Jeddah est considérée comme la principale porte d'entrée terrestre et maritime des pèlerins à destination de la ville sainte de la Mecque, qui arrivaient exclusivement par voie maritime et terrestre.

Le plus grand changement dans la fonction de la ville de Jeddah a eu lieu en 647 après JC, lorsque le calife musulman l'a choisie pour être le principal port pour l'entrée à La Mecque, en lieu et place du port de Shuaiba, qui est situé plus au sud (EMCC, 2017). Par conséquent, l'activité portuaire est devenue la fonction principale de la ville, qui a acquis une dimension politique importante lorsqu'elle a officiellement rejoint le Royaume d'Arabie Saoudite en 1925. Après la découverte du pétrole en 1938, Jeddah est devenue la capitale de la région ouest, et donc la position administrative est devenue la caractéristique dominante de la ville, ce qui s'est traduit par la fourniture de services publics, l'extension des routes et la liaison avec le reste des autres villes avec un réseau de transport solide.

Jeddah a connu une croissance urbaine sans précédent en raison de multiples facteurs dont le développement économique, l'amélioration des services et offres de divertissement, l'augmentation de la population qui était estimée à environ 1 million d'habitants en 1970, et a dépassé les 3 millions en 2010 -(Ewea, 2010, Aljaddani, 2105, Almazroui et al., 2017). La ville s'est étendue principalement sur la plaine côtière de la mer Rouge dont le littoral subit une très forte pression, allant jusqu'à l'édification de terre-pleins sur la mer où sont installés divers infrastructures et équipements publiques ou privés.

L'objectif principal de notre étude est le suivi diachronique de ces étendues artificielles de terres acquises sur la mer, par l'utilisation de données de télédétection. Il s'agit de mesurer la surface de ces terres et leurs affectations urbaines et d'en suivre les implications environnementales et morphologiques, entre 1965 et 2020. Cette ville étant la plus grande agglomération portuaire la mer Rouge (Spalding et al.2001), son littoral est un espace très convoité et entièrement artificialisé, d'une part à cause de la croissance urbaine et démographique de la ville et, d'autre part, en raison du cadre 
géomorphologique de la cité coincée entre les montagnes du Hedjaz à l'est et la mer Rouge à l'ouest (Abu Ouf, M. and EI-Shater, 1991 ; Al-Sheikh, 2011, Youssef et al.2016).

Il apparaît ainsi intéressant d'évaluer les tendances historiques se rapportant aux zones urbaines en bordure de mer en termes de superficie et d'affectation du sol, par l'utilisation de données de télédétection et les systèmes d'information géographique. Une cartographie et une analyse statistique et spatiotemporelle de la dynamique et de l'utilisation des terre-pleins et leurs impacts environnementaux seront réalisés. L'étude montre également l'importance de l'imagerie satellite CORONA pour les études urbaines et environnementales multitemporelles car elle constitue une importante source de données de télédétection historiques à très haute résolution et à large couverture spatiale.

\section{2- La zone d'étude}

La ville de Jeddah est située au centre-ouest du royaume d'Arabie Saoudite, sur la côte occidentale de la mer Rouge, dans une zone aride de la région d'Hedjaz. La ville s'entend actuellement sur environ $100 \mathrm{~km}$ le long de la plaine littorale de la Tihamah avec une largeur moyenne d'environ $25 \mathrm{~km}$ (figure 1).

La ville été choisie pour de nombreuses raisons afin de mettre en œuvre cette étude. Elle est la deuxième plus grande ville du royaume d'Arabie Saoudite par sa taille et sa population, après la capitale Riyad. Elle est cosmopolite et constitue le plus grand centre urbain du littoral de la mer Rouge dont elle abrite le plus ancien et le plus grand port en termes de volume de marchandises et de trafic de passagers. Ceci a contribué à faire de Jeddah l'une des villes commerciales les plus importantes du Royaume (Abdulghani, 1983). Sa situation géographique sur les anciennes routes commerciales et son statut de port maritime et d'aéroport par lesquels transite la grande majorité des pèlerins se rendant dans la ville sainte de la Mecque, a valu à la ville le plus grand essor en Arabie Saoudite. 


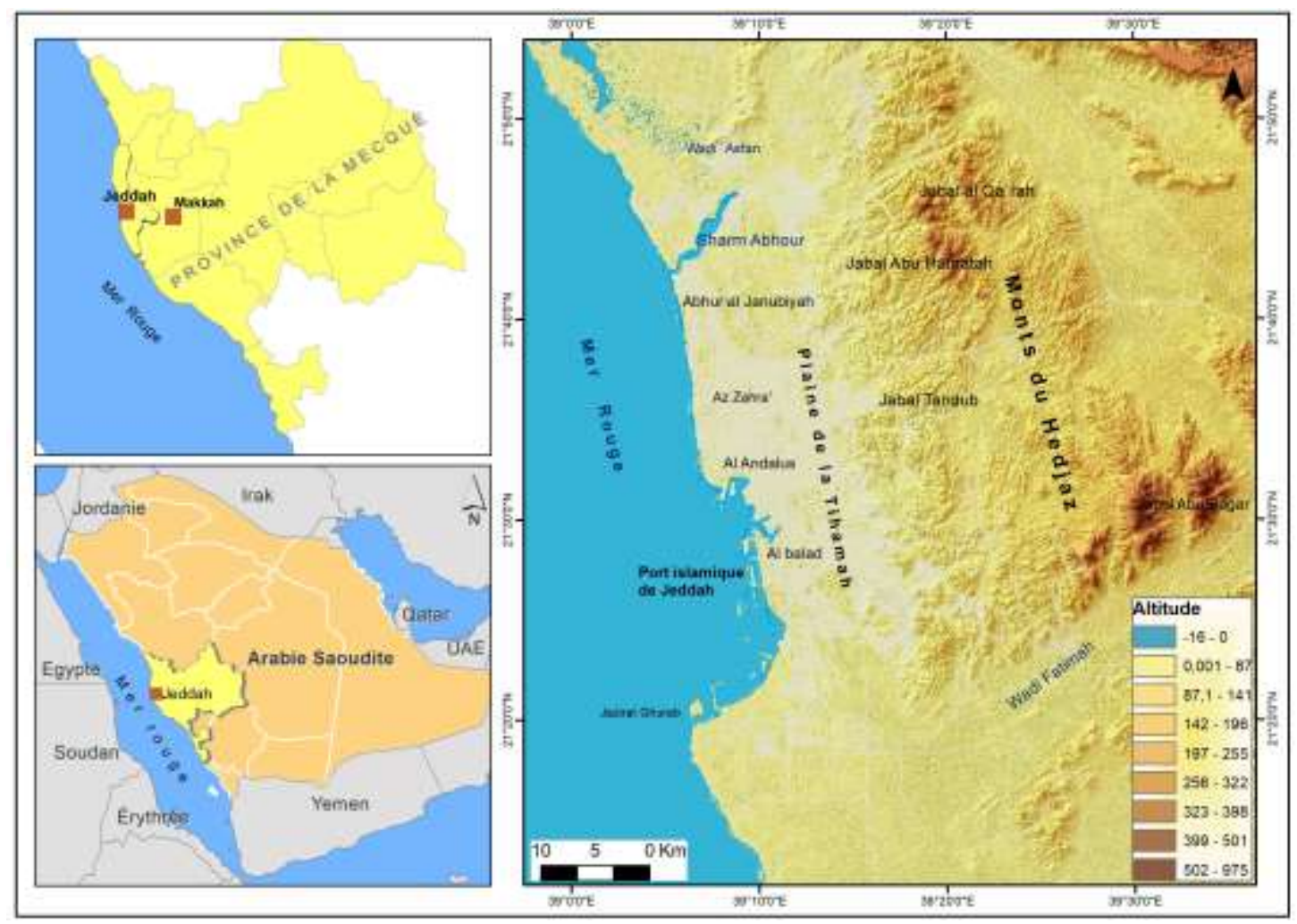

Figure 1. La zone d'étude

Jeddah a commencé à acquérir plus d''importance au XXème siècle, lors de la croissance économique consécutive au second boom pétrolier des décennies 70 et 80 (Abdu et al. 2002 ; Helmi. 2015). Selon Abdu et al. (2002). Cette période marque le développement économique et diplomatique de la ville de Jeddah, ce qui fait dire à Peter (2003) qu'à bien des égards, Jeddah est la réussite de l'Arabie saoudite.

La ville compte environ 4 millions de personnes, soit 13,4\% de la population totale du Royaume (selon les estimations de l'année 2018). L'essor de la ville apparait aussi au niveau régional, elle intègre 20,3 . \% du total des travailleurs étrangers au royaume d'Arabie Saoudite, ce qui signifie que la moitié de la population de la ville est constituée d'immigrés, selon le recensement de 2010.

Par ailleurs, l'extension de la ville sur le cours et l'exutoire de plusieurs oueds descendant des montagnes du Hedjaz, entraine des inondations catastrophiques lors des fortes et intenses précipitations qui tombent dans cette zone malgré l'aridité des conditions climatiques (Daoudi et Niang, 2019). Cette croissance urbaine vers la mer 
entraine également la disparition de plusieurs ilots notamment dans la baie de Jeddah et des perturbations de l'environnement marin (Mansour et Madkour, 2015).

\section{3- Données utilisées et méthodologie}

\subsection{Données utilisées}

. L'imagerie satellitaire à haute résolution spatiale est une source essentielle pour étudier les changements historiques de phénomènes en évolution rapide, grâce à la disponibilité d'informations spatiales sur une zone en différentes périodes.

Les données de télédétection multisources et multitemporelles à très haute résolution spatiale ont été utilisées dans cette étude pour couvrir la ville de Jeddah en 1965, 1972, 1986, 2003, et 2020. Les deux premières années sont couvertes par des photographies satellites de la mission CORONA. L'importance des images Corona tient au fait qu'elles constituent de rares données de télédétection avec une très haute résolution spatiale disponibles pour les années 60 et 70 . Les autres périodes sont couvertes par des images satellites optiques ; SPOT CIB (1986), OrbView3 (2003) et Sentinel-2 (2020). Toutes les données satellites ont été obtenues à partir du site web de l'USGS (https://earthexplorer.usgs.gov) et ont une résolution spatiale comprise entre 1 et $10 \mathrm{~m}$. Elles sont également en mode panchromatique sauf celle de 2020. Les détails des images sont consignés dans le tableau 1.

Tableau 1 : Details des images utilisées

\begin{tabular}{|c|c|c|}
\hline Type de données & Date d'acquisition & Résolution (m) \\
\hline CORONA/Satellite photo. & $05 / 03 / 1965$ & 3 \\
\hline CORONA/ Satellite photo. & $27 / 05 / 1972$ & 2 \\
\hline SPOT CIB 10 & $24 / 031986$ & 10 \\
\hline Orbview-3 & $05 / 12 / 2003$ & 1 \\
\hline Sentinel 2 & $27 / 03 / 2020$ & 10 \\
\hline
\end{tabular}

Par ailleurs des couches de données vectorielles ont été utilisées, elles ont été extraites des images satellites par digitalisation. Il s'agit essentiellement pour chaque année; extraction du trait de côte, délimitation du bâti urbain, identification de l'occupation du sol en bord de mer. Une base de données SIG a été réalisée permettant la cartographie de la croissance urbaine et de l'occupation et l'utilisation des sols entre 1965 et 2020. 


\subsection{Traitement des images}

La superposition correcte des images multidates et multisources requiert une correction géométrique et un géoréférencement. Le processus de correction géométrique est effectué à partir des points de contrôle sur l'image de référence à partir de laquelle toutes les autres ont été rectifiées (le nombre dépend des images à corriger, le plus grand nombre de points a été sélectionné sur les photographies satellite Corona à cause des distorsions dont elles font l'objet).L'image Sentinel-2 qui est orthorectifiée a été choisie comme référence La correction géométrique est effectuée à l'aide du logiciel ERDAS IMAGINE par la méthode polynomiale, afin d'éliminer les distorsions liées à la variation d'échelle, à l'inclinaison et à la distorsion de l'objectif. Après rectification, la création des couches vectorielles a été effectuée avec le logiciel ArcGIS version 10.7 .

\subsection{Evaluation de l'extension urbaine des zones côtières}

Jeddah est l'une des villes qui a connu les plus grands changements urbains au niveau des zones côtières du Royaume. Afin d'évaluer la superficie des terres édifiées sur la mer et l'affectation des sols, plusieurs techniques sont adoptées :

- Cartographie des lignes de rivages à partir des images satellites sous ArcGIS 10 et déduction de l'évolution des surfaces artificialisées (négatives ou positives) entre deux dates successives par les outils d'analyse spatiale.

- Cartographie de l'utilisation des sols sur les surfaces obtenues à partir de deux images successives.

- Analyse statistique détaillée des superficies des terre-pleins et de leurs occupations calculées pour les différentes années et suivi de leur rythme de croissance.

- Etude des impacts géomorphologiques et environnementaux liés à l'extension urbaine dans les zones côtières

- Etude et analyse de différents aspects et approches liés à l'urbanisation (aspects fonctionnels de la ville, morphologie urbaine). 


\section{Résultats}

\subsection{Extension urbaine de Jeddah au détriment de la mer}

Le remblaiement de zones côtières de la mer Rouge à Jeddah et leur annexion au tissu urbain n'ont pas cessés depuis 1965. La surface totale de terres gagnées sur la mer (terre-pleins) sur la période d'observation a atteint $32,8 \mathrm{~km}^{2}$, ce qui signifie que chaque année, durant la période d'étude, $597148 \mathrm{~m}^{2}\left(0,59 \mathrm{~km}^{2}\right)$ de mer ou d'ilots ont été transformés en secteurs urbains. Le tableau 2 et la figure 2 montrent les étapes de l'extension urbaine au détriment de la mer entre 1965 et 2020.

Tableau 2. Evolution de la croissance urbaine au détriment de la mer entre 1965 et 2020

\begin{tabular}{|c|c|c|c|c|}
\hline Période & $\begin{array}{c}\text { Superficie } \\
\text { remblayée } \mathbf{k m}^{2}\end{array}$ & $\mathbf{\%}$ & $\begin{array}{c}\text { Croissance moyenne } \\
\text { annuelle } \mathbf{k m}^{2}\end{array}$ & $\mathbf{\%}$ \\
\hline $1965-1972$ & 1,54 & 4,7 & 0,22 & 0.7 \\
\hline $1972-1986$ & 24,51 & 74,7 & 1,75 & 5,3 \\
\hline $1986-2003$ & 3,69 & 11,2 & 0,21 & 0,7 \\
\hline $2003-2020$ & 3,08 & 9,4 & 0,18 & 0,6 \\
\hline Total & $\mathbf{3 2 , 8 4}$ & $\mathbf{1 0 0}$ & $\mathbf{0 , 5 9}$ & $\mathbf{1 , 8}$ \\
\hline
\end{tabular}

Il ressort de l'analyse des données que la première période (1965-1972) a enregistré la plus faible croissance urbaine au détriment de la mer, seule une superficie équivalente à $1,54 \mathrm{~km} 2$ de terre-plein a été édifiée, soit 4,7\% de la surface totale de l'expansion urbaine au détriment de la mer.

Les années 70 et 80 étant celles de l'explosion démographique et urbaine de la ville de Jeddah, ce fut la période de la plus forte expansion spatiale au détriment de la mer, où environ $75 \%$ des terres acquises sur la mer ont été aménagées (entre 1972 et 1986). La zone maritime a perdu $24,5 \mathrm{~km} 2$ sous la pression de l'urbanisation. L'accroissement moyen annuel au cours de cette période était de 1,75 km2.

Lors de la période 1986 - 2003, la part de l'expansion urbaine au détriment de la mer était de 3,69 km2 soit 11,2\%, le taux le plus élevé après celui de la période précédente.

La construction de terre-pleins a ensuite diminué au cours de la dernière période (2003-2020) où environ $3 \mathrm{~km}$ de nouvelles terres ont été acquises sur la mer, soit 9,4\% de la superficie totale sur toute la période. 


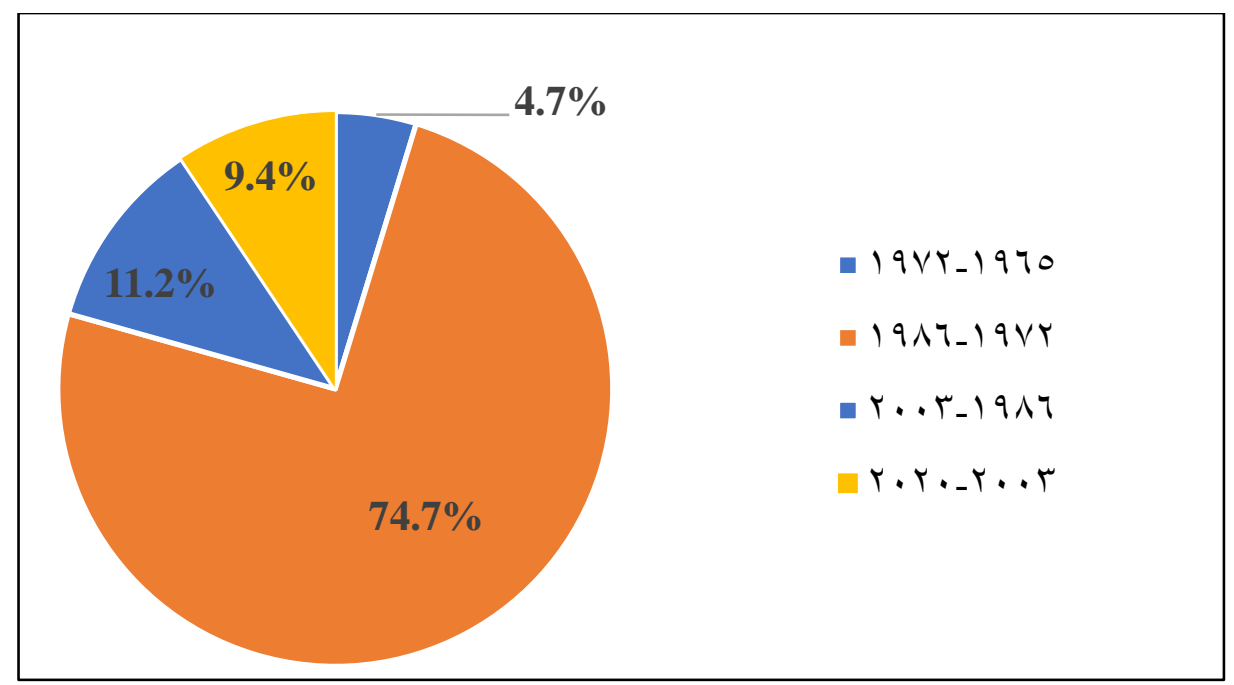

Figure2. Évolution du taux de croissance urbaine au détriment de la mer

L'utilisation détaillée des terres aménagées lors des différentes périodes permet de connaître les véritables raisons de l'expansion urbaine au détriment de la mer à chaque étape. Toutes les terres n'étaient pas à usage unique ou au service d'une activité spécifique, mais leur usage variait à chaque période et la proportion de chaque activité changeait également en fonction du contexte socio-économique et des objectifs et orientations spécifiques. Le port de Jeddah a bénéficié de la plus grande zone d'expansion aux dépens de la mer pendant la période d'étude. Cette extension spatiale du port a atteint environ $11,85 \mathrm{~km} 2$ soit $36,1 \%$ de la superficie totale des terre-pleins. Il ressort du tableau 3 que les infrastructures administratifs et gouvernementaux constituent la deuxième plus importante affectation des sols; il s'agit de services, d'institutions et de directions publics ou logistiques. Ces services administratifs occupent une superficie de $7.05 \mathrm{~km} 2$ soit $21,5 \%$ du total des surfaces récupérées sur la mer au niveau de la zone.

Tableau 3. Surface et affectations des sols sur différentes périodes

\begin{tabular}{|c|c|c|c|c|c|c|c|c|c|c|}
\hline \multirow{2}{*}{ Utilisation du sol } & \multicolumn{2}{|c|}{$1965-1972$} & \multicolumn{2}{|c|}{$1972-1986$} & \multicolumn{2}{c|}{$1986-2003$} & \multicolumn{2}{c|}{$2003-2020$} & \multicolumn{3}{c|}{ Total } \\
\cline { 2 - 12 } $\mathrm{km}^{2}$ & $\%$ & $\begin{array}{c}\text { Surface } \\
\mathrm{km}^{2}\end{array}$ & $\%$ & $\begin{array}{c}\text { Surface } \\
\mathrm{km}^{2}\end{array}$ & $\%$ & $\begin{array}{c}\text { Surface } \\
\mathrm{km}^{2}\end{array}$ & $\begin{array}{c}\% \\
\mathrm{Km}^{2}\end{array}$ & $\begin{array}{c}\text { Surface } \\
\%\end{array}$ \\
\hline Port & 1,51 & 97,9 & 8,94 & 36,5 & 0.89 & 24 & 0.50 & 16,2 & 11.85 & 36,1 \\
\hline Gouvernemental & 0,03 & 2,1 & 5.87 & 24 & 0,23 & 6,3 & 0,91 & 29,7 & 7.05 & 21,5 \\
\hline Service Public & - & - & 3,86 & 15,7 & 0,01 & 0,2 & 1,52 & 49,4 & 5,39 & 16,4 \\
\hline Touristique & - & - & 2,31 & 9,4 & 2,54 & 69 & 0.15 & 4,7 & 5.00 & 15,2 \\
\hline $\begin{array}{c}\text { Résidentiel/ } \\
\text { Commercial }\end{array}$ & - & - & 3.53 & 14,4 & 0.02 & 0,5 & - & - & 3.55 & 10,8 \\
\hline Total & 1,54 & 100 & 24,51 & 100 & 3,69 & 100 & 3,08 & 100 & 32,84 & 100 \\
\hline
\end{tabular}




\section{4-2 . Extension urbaine et utilisation du sol au Port de Jeddah}

La superficie totale du port de Jeddah est actuellement de $12,5 \mathrm{~km}^{2}$. Cette surface englobe un terre-plein d'environ $11,85 \mathrm{~km}^{2}$ soit $94,4 \%$ de la zone industrialo-portuaire, construit en diverses phases, au cours de la période d'étude. L'extension du port par remblaiements successifs est illustrée à la figure 3 .
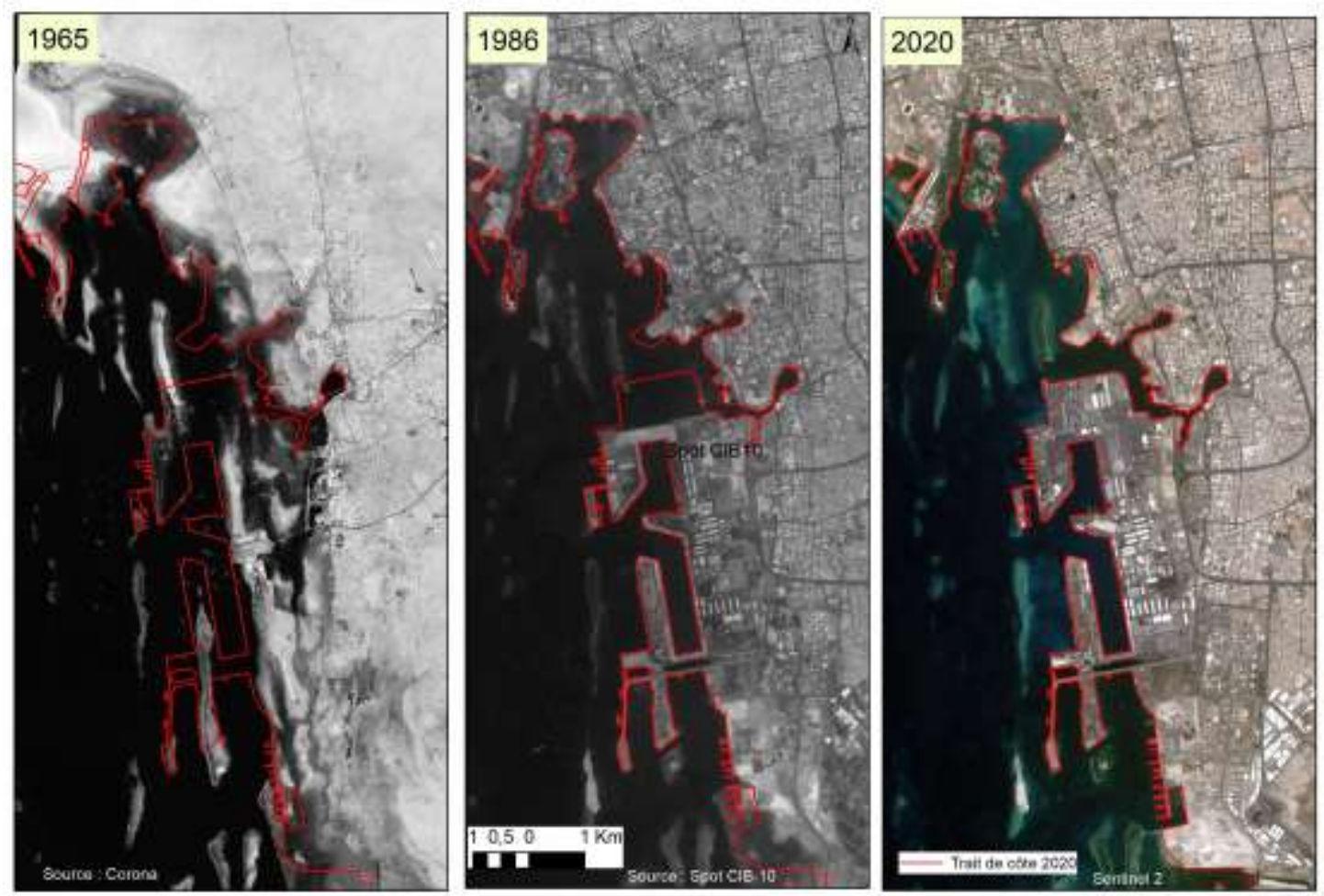

Figure 3. Illustration de trois phases d'extension du port par remblaiements successifs

De façon générale, la zone portuaire de Jeddah réunit le pourcentage le plus élevé d'extension urbaine gagnée sur la mer, soit 36\% des terre-pleins de la ville. En effet, au cours des 55 dernières années de la période d'étude, le port n'a cessé de s'élargir au détriment de la mer.

Afin d'estimer l'importance et le poids économique du port de Jeddah, on peut souligner qu'en 2019, le tonnage de marchandises déchargé et chargé a atteint 55,2 millions de tonnes, ce qui représente $21 \%$ du total des importations et exportations de marchandises du Royaume d'Arabie Saoudite. Le port de Jeddah revêt également une importance particulière liée à sa dimension historique, car il fut la principale porte d'entrée pour le pèlerinage vers les lieux Saints de l'Islam (Mecque et Médine) à travers les âges. C'est le principal port qui reçoit les fidèles en provenance de toutes les parties du monde, il a accueilli près d'un demi-million de passagers (entrants ou sortants), ce 
qui équivaut à $36 \%$ du total de trafic maritime de passagers transitant dans les neuf ports saoudiens, que ce soit sur la mer Rouge ou le golfe Persique.

Le tableau 4 et la figure 4, indiquent que la période qui a enregistré la plus grande expansion du port aux dépens de la mer a été celle de 1972 à 1986 qui totalise à elle seule $75.5 \%$ de la superficie globale des terre-pleins du port édifiés durant toute la période d'étude. Cette grande extension du port peut être justifiée par le contexte socioéconomique du royaume. Cette période est marquée par une croissance économique attribuée au second boom pétrolier 1974-1986 (Daghistani, 1993 ; Abdu et al. 2002 ; Helmi. 2015). Le port a enregistré sa plus grande extension spatiale matérialisée par un développement sans précèdent des infrastructures urbaines et installations portuaires (Daghistani, 1993, Helmi, 2015). Cette phase marque l'essor économique et diplomatique de Jeddah qui a acquis un rôle important au niveau national et international. Plusieurs projets et programmes de développement dont un plan Directeur ont été adoptés avec une priorité au secteur urbain (Abdu et al. 2002).

La première période entre 1965 et 1972, bien qu'étant la plus courte de toutes, révèle une augmentation considérable de la superficie du port par remblaiement, qui totalise $12,8 \%$ de la surface totale gagnée sur la mer sur toute la période d'observation. On assiste lors de cette étape au début du développement économique et à la construction et l'exploitation de navires pour le transport maritime, afin de répondre à l'augmentation du nombre de fidèles arrivant pour le pèlerinage à la Mecque, qui se situe à environ $85 \mathrm{~km}$ de Jeddah.

Le tableau 4 indique également que toutes les sous-périodes dévoilent une augmentation constante de la superficie du port, avec une moyenne de $0,21 \mathrm{~km}^{2}$ par année

Tableau 4. Augmentation de la superficie du port pour différentes périodes

\begin{tabular}{|c|c|c|c|c|}
\hline Période & Extension $\mathbf{k m}^{\mathbf{2}}$ & $\mathbf{\%}$ & Moyenne annuelle $\mathbf{~ k m}^{\mathbf{2}}$ & $\mathbf{\%}$ \\
\hline $1965-1972$ & 1,51 & 12.8 & 0,21 & 1.8 \\
\hline $1972-1986$ & 8,94 & 75.5 & 0,63 & 5.4 \\
\hline $1986-2003$ & 0,89 & 7.5 & 0,05 & 0.4 \\
\hline $2003-2020$ & 0,50 & 4.2 & 0,03 & 0.2 \\
\hline Total & $\mathbf{1 1 , 8 5}$ & $\mathbf{1 0 0}$ & $\mathbf{0 , 2 1}$ & $\mathbf{1 . 8}$ \\
\hline
\end{tabular}




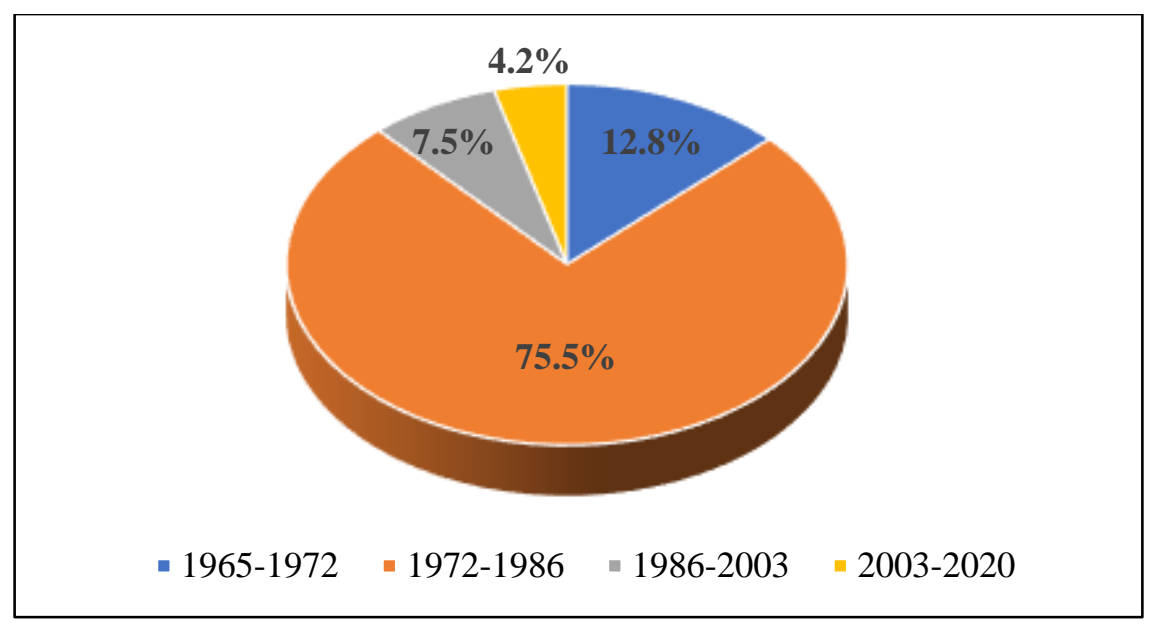

Figure 4. Taux d'extension de la surface du port au détriment de la mer à différentes périodes

Cette grande extension de la zone portuaire s'est accompagnée du développement de nombreuses activités logistiques et la construction d'infrastructures et d'équipements diverses sur le site du port au cours de cette période, il s'agit de terminaux à conteneurs ou pour le bétail ou de silos à grains et autres installations que l'on peut voir sur la figure 5 .

Entre 1986 et 2003, le port s'est agrandi d'une superficie de $0,89 \mathrm{~km}^{2}$, équivalant à $7.5 \%$ de la proportion de la zone d'expansion aux dépens de la mer à Jeddah. Des agrandissements ont eu lieu au nord du port et ont servi à élargir le terminal à conteneurs du nord. La période de 2003 à 2020 enregistre la plus faible expansion du port, malgré une gradation de $0,5 \mathrm{~km}^{2}$ soit $4,2 \%$ du total des terre-pleins. Les extensions ont eu lieu au nord et au sud du port et ont servi à l'agrandissement du terminal à conteneurs et à la construction de nouveaux quais, en particulier au sud du port.

\subsection{Utilisation des sols dans les autres zones acquises sur la mer}

D'autres services publics (routes, jardins, plages publiques, etc.) occupent d'environ $5,39 \mathrm{~km}^{2}$, soit $16,4 \%$ des terre-pleins. Alors que l'utilisation touristique (représentée par les hôtels, les plages et les clubs privés) occupe 15,2\% de l'expansion totale sur la mer. Les utilisations mixtes résidentielles et commerciales totalisent 10,8\% de l'expansion totale aux dépens de la mer. Tous les types d'utilisation des sols ont enregistré leur plus important taux lors de la période 1972-1986 sauf l'usage touristique qui a atteint son maximum à partir de la période 1986-2003, Cela s'explique par la nouvelle orientation vers le tourisme littoral (Figure 6) 


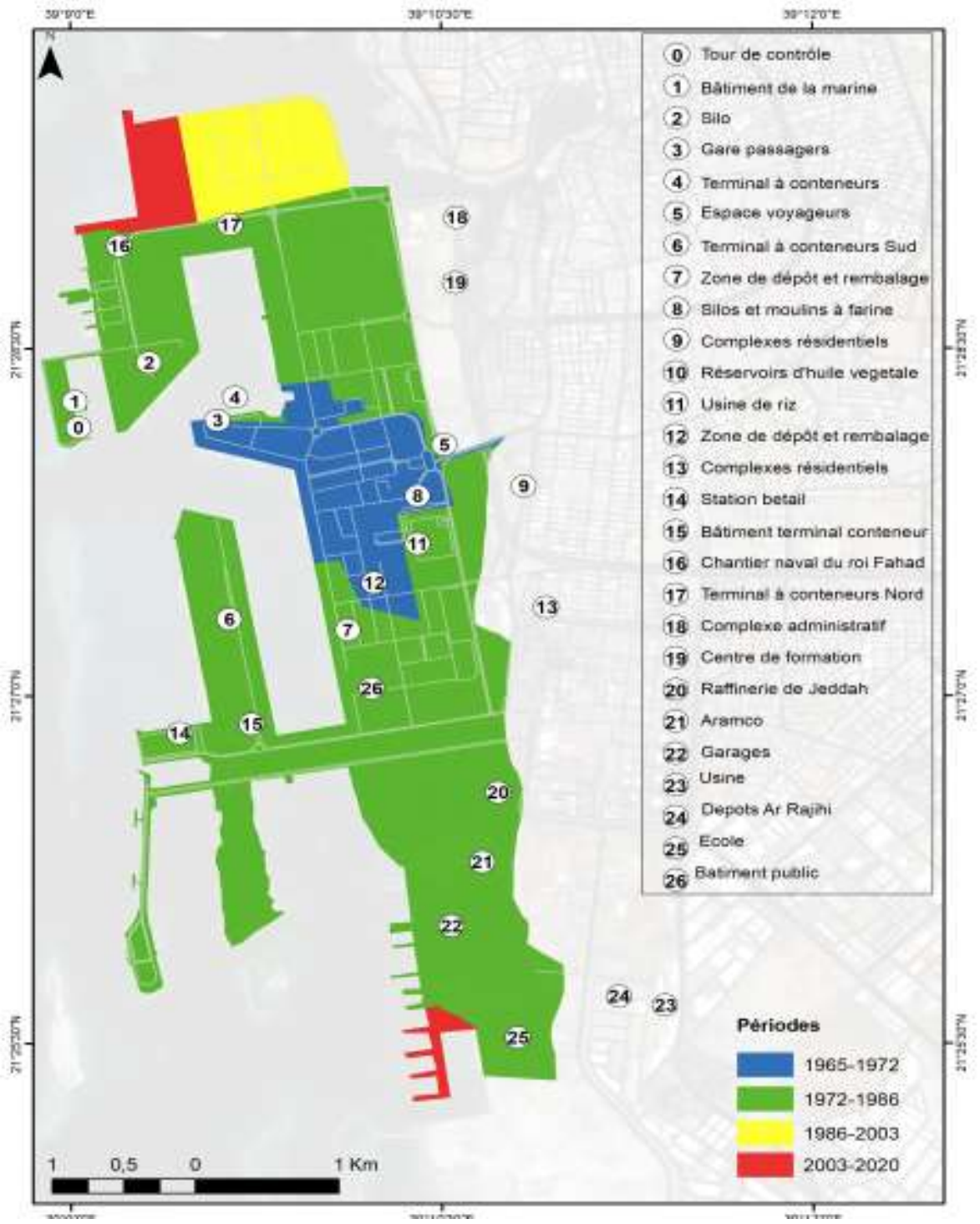

Figure 5. Les différentes phases d'édification des terre-pleins et d'occupation des sols au port islamique de Jeddah

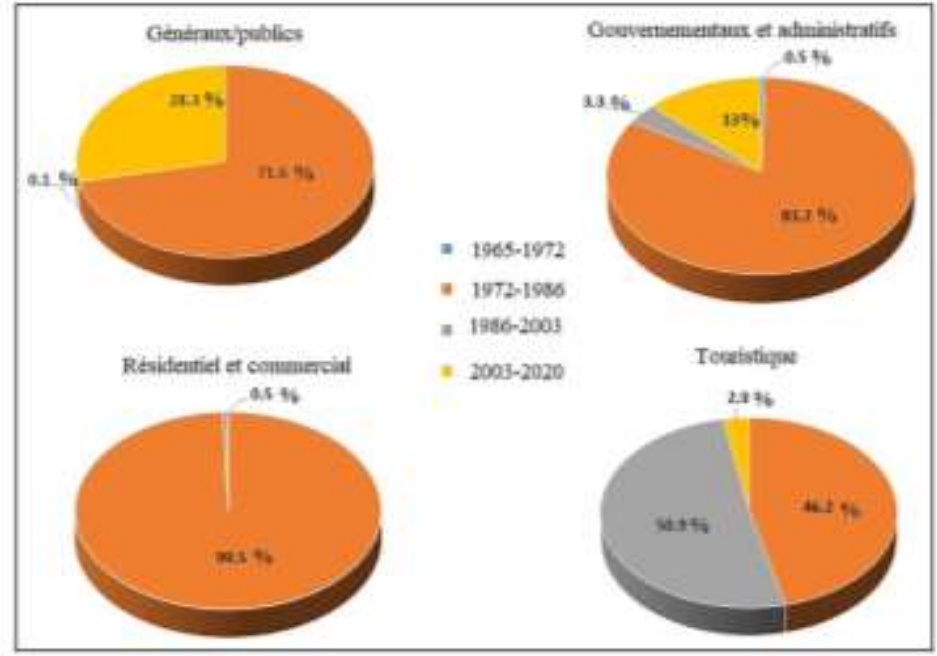

Figure 6. Taux des différents types d'utilisations du sol en fonction des périodes 


\section{- Utilisation gouvernementale et administrative des sols :}

La superficie des terres utilisées pour les activités gouvernementales a été variable tout au long de la période d'étude. La période où la plus forte augmentation de ces terres a été observée est celle entre 1972 et 1986. Au cours de cette phase, les usages des terres pour les activités gouvernementales se sont étendus sur une superficie de $5,87 \mathrm{~km}^{2}$, soit $24 \%$ de l'utilisation totale des terres au cours de la période étudiée. Ces affectations étaient diverses et variées, il s'agissait de complexes de logements pour les employés de certaines institutions gouvernementales, des écoles affiliées à ces complexes et des mosquées. Il y avait également des usines de dessalement d'eau de mer, qui produisent aussi de l'électricité, des usines de traitement des eaux usées dans le district d'Al Balad et des stations de bus de transport en commun. Ces agrandissements comprenaient la construction de la municipalité de Jeddah et le principal marché aux poissons.

\section{- Utilisation générale/publique :}

La période où la plus grande surface de terres gagnées sur la mer a été affectée à l'usage public était celle entre 1972 et 1986 avec une superficie de 3,86 km², équivalant à 71,6\% des utilisations publiques totales, suivie de la période $2003-2020$ où $1,5 \mathrm{~km}^{2}$ était utilisé soit $28,3 \%$ des usages publiques. Les utilisations publiques étaient représentées par les plages publiques gratuites, la promenade maritime privée pour les pique-niques, les parcs et les routes (voir figure 7) 


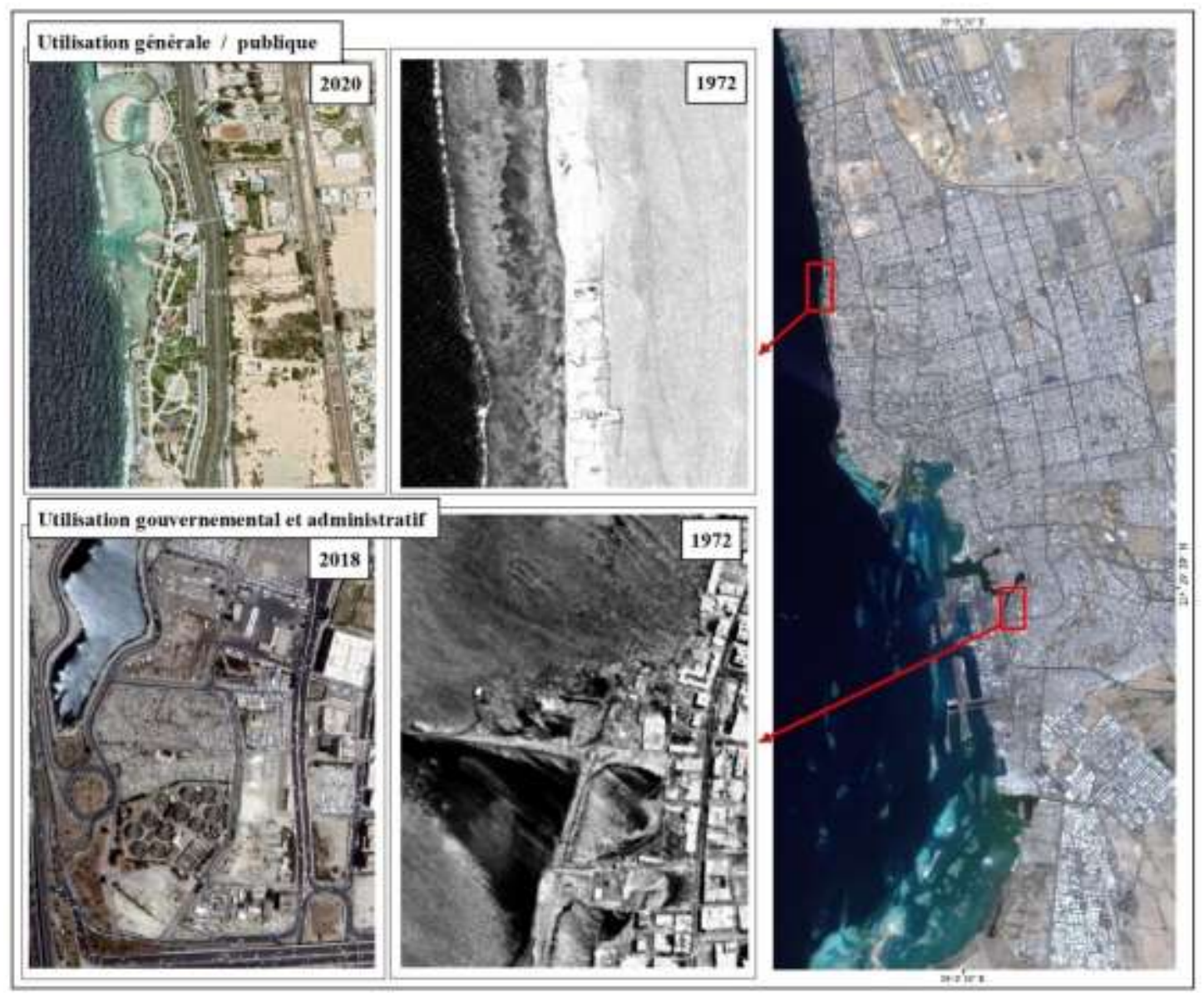

Figure 7. Illustration de l'utilisation gouvernemental, administratif/ publique des sols

\section{- Utilisation touristique :}

Le début d'exploitation des terre-pleins à des fins touristiques a eu lieu entre 1972 et 1986, lorsque 2,31 $\mathrm{km}^{2}$ des nouvelles terres ont été dédiés à cette activité, soit 46,2\% $\mathrm{du}$ total des usages touristiques. La superficie des infrastructures touristiques a ensuite augmenté pour atteindre $2,54 \mathrm{Km}^{2}$ entre 1986 et 2003 soit 50,9\% du total des usages touristiques. Les utilisations touristiques sont présentes dans les plages privées, les hôtels et restaurants, la pêche, les bateaux touristiques et les stations balnéaires, en particulier dans le nord de Charm Abhur (Figure 8) 


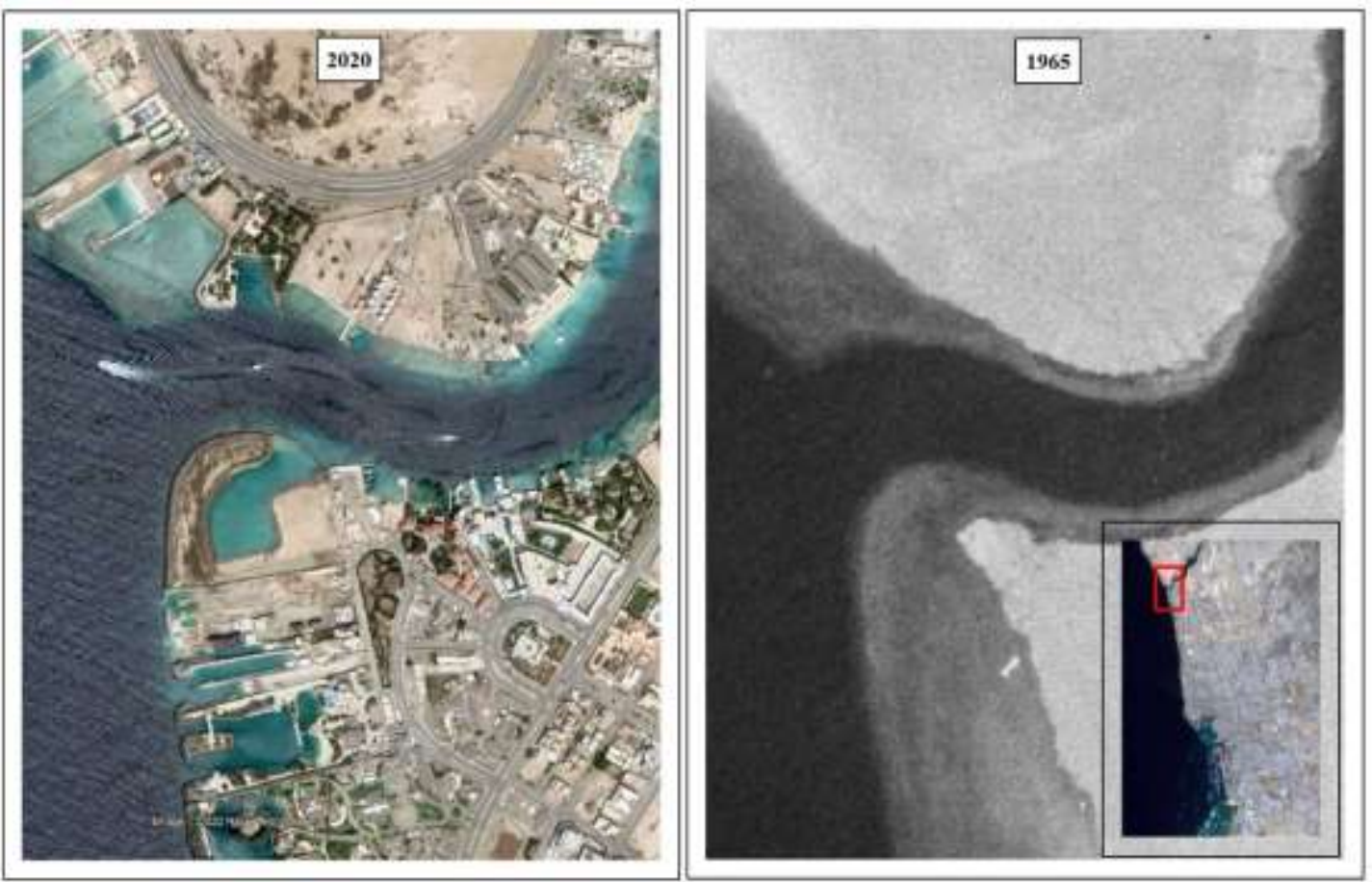

Figure 8. Exemple d'utilisation touristique des sols dans la zone de Sharm Abhur

\section{- Utilisation résidentielle et commerciale :}

Ce type d'usage est le moins présent dans les superficies gagnées sur la mer car l'usage résidentiel peut s'étendre dans des espaces disponibles à la périphérie de la ville de Jeddah et non dans la zone centrale. Cet usage a occupé, $3,53 \mathrm{~km}^{2}$ de la côte, sur la période 1972 -1986 représentant 99,5\% du total de ce type d'utilisation. Il était principalement concentré à Al-Balad et Al-Andalus, qui font partie des plus anciens quartiers de la ville de Jeddah (Sahahiri et al 2019), et ce type d'utilisation se caractérise par un mélange entre logements, bureaux, institutions privées et magasins Commerciaux (figure 9) 


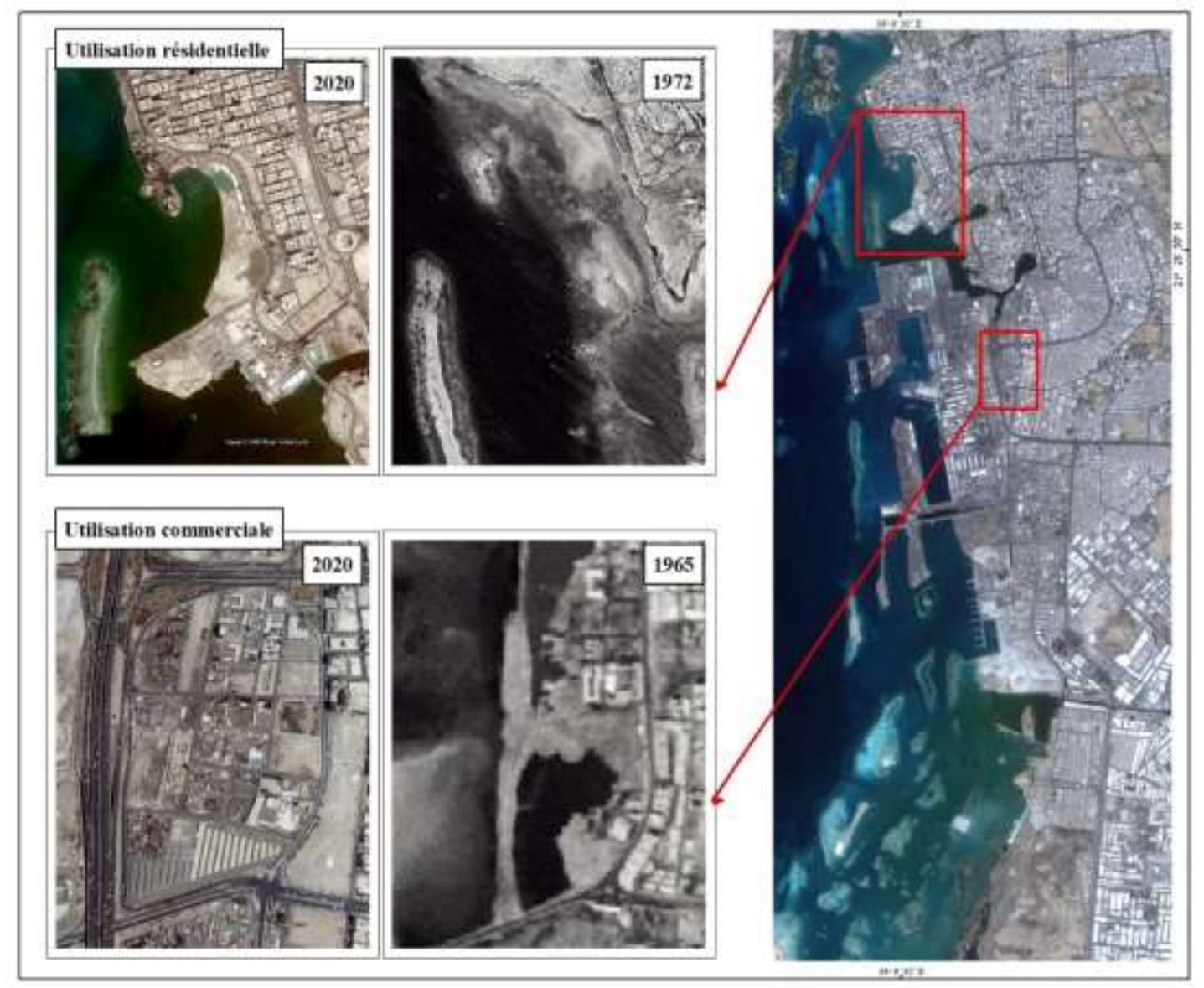

Figure 9. Illustration de l'utilisation résidentielle et commerciale des sols

\subsection{Impact environnementaux et morphologiques de l'extension urbaine dans la zone côtière}

Les changements apportés à la configuration du littoral ont aussi concerné l'environnement côtier. Les écosystèmes et habitats marins de la zone proche du rivage ont été affectés. Une déplétion de la superficie des mangroves ainsi que leur dégradation ont été constatée dans la partie sud du littoral par Al-Dubai et al. (2017) qui les ont attribués aux activités anthropogéniques dans la zone côtière. Les aménagements du littoral ont provoqué la destruction de la vie maritime en étouffant les communautés benthiques et réduisant les rendements et les stocks de poissons, selon Tortel (2004), qui note aussi des problèmes de pollutions. Selon Mansour et Madkour (2015) la plupart des grands ports et sites touristiques dans différents pays le long de la mer Rouge sont construits sur des récifs coralliens (voir figures 8 et 9). Ces mêmes auteurs affirment qu'une partie de l'autoroute vers le nord de Jeddah est construite sur un platier récifal, ce qui entraine de graves pertes 
d'habitats côtiers. La figure 10 montre la construction d'une route sur le récif corallien au sud du port et la liaison de l'ile de Ghurab au continent par remblaiement.
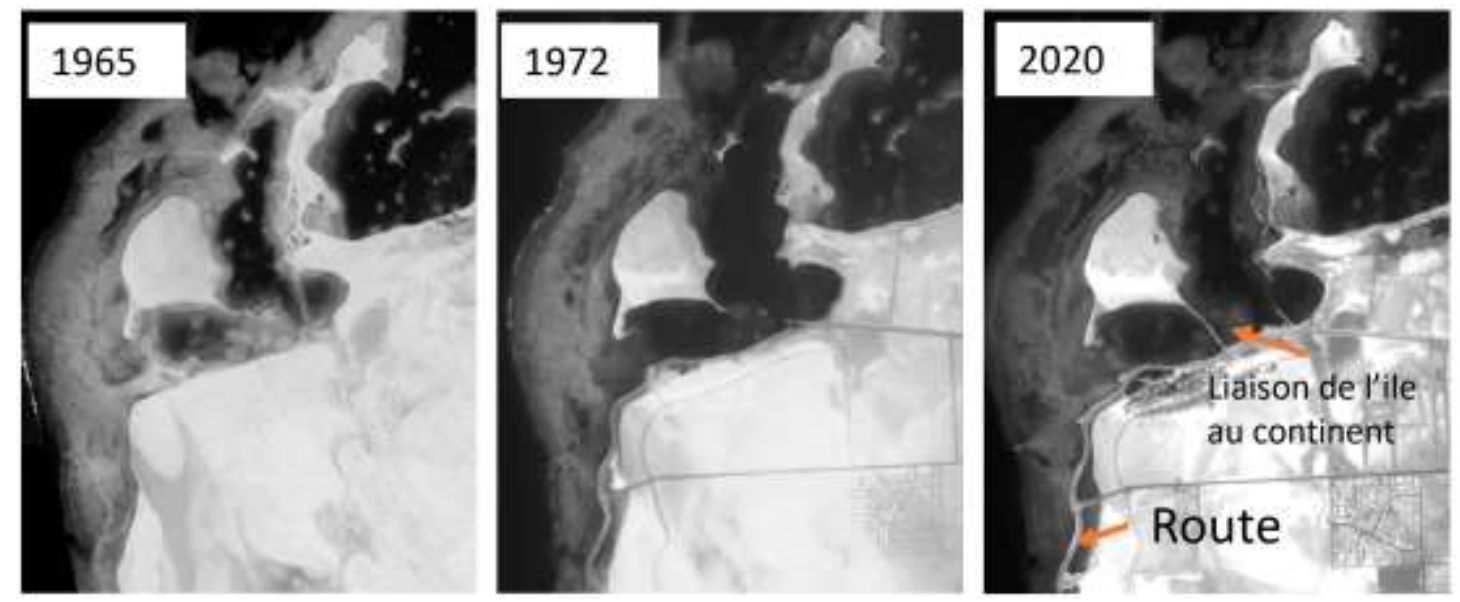

Figure 10. Changements dans la configuration du littoral au sud du port entre 1965 et 2020 ; jonction de l'ile de Ghurab au continent et construction d'une route littorale sur un récif

La géomorphologie du littoral a été également modifiée. Les rivages de Jeddah sont à côtes meubles qui peuvent subir rapidement des phases d'accrétion et d'érosion. La Figure 11 illustre quelques-uns des nombreux changements environnementaux dans la zone côtière entre 1965 et 2020 : disparition ou apparition d'iles et d'ilots, disparition de récifs coralliens, dragages ou remblaiements de la mer pour les besoins d'infrastructures touristiques ou gouvernementaux.

Les différents travaux d'extension du port mettent en évidence un élargissement de la plaine côtière impliquant la création de lagunes semi-fermées le long du littoral et la disparition de plusieurs récifs et ilots surtout dans la zone portuaire (figure 11. 2B). L'urbanisation du littoral l'extraction, le dragage et le déplacement des sables du littoral a aussi comme conséquence un changement de la morphologie de la ligne de rivage (Luijendijk et al. 2018). La figure 8 illustre ce changement de la morphologie du littoral sur quelques sites entre 1965 et 2020. Le rivage qui avait une forme plus ou moins rectiligne en 1965 (Figure 11.1A) présente plusieurs sinuosités suite aux différentes installations sur la plage (Figure 11.1B) 


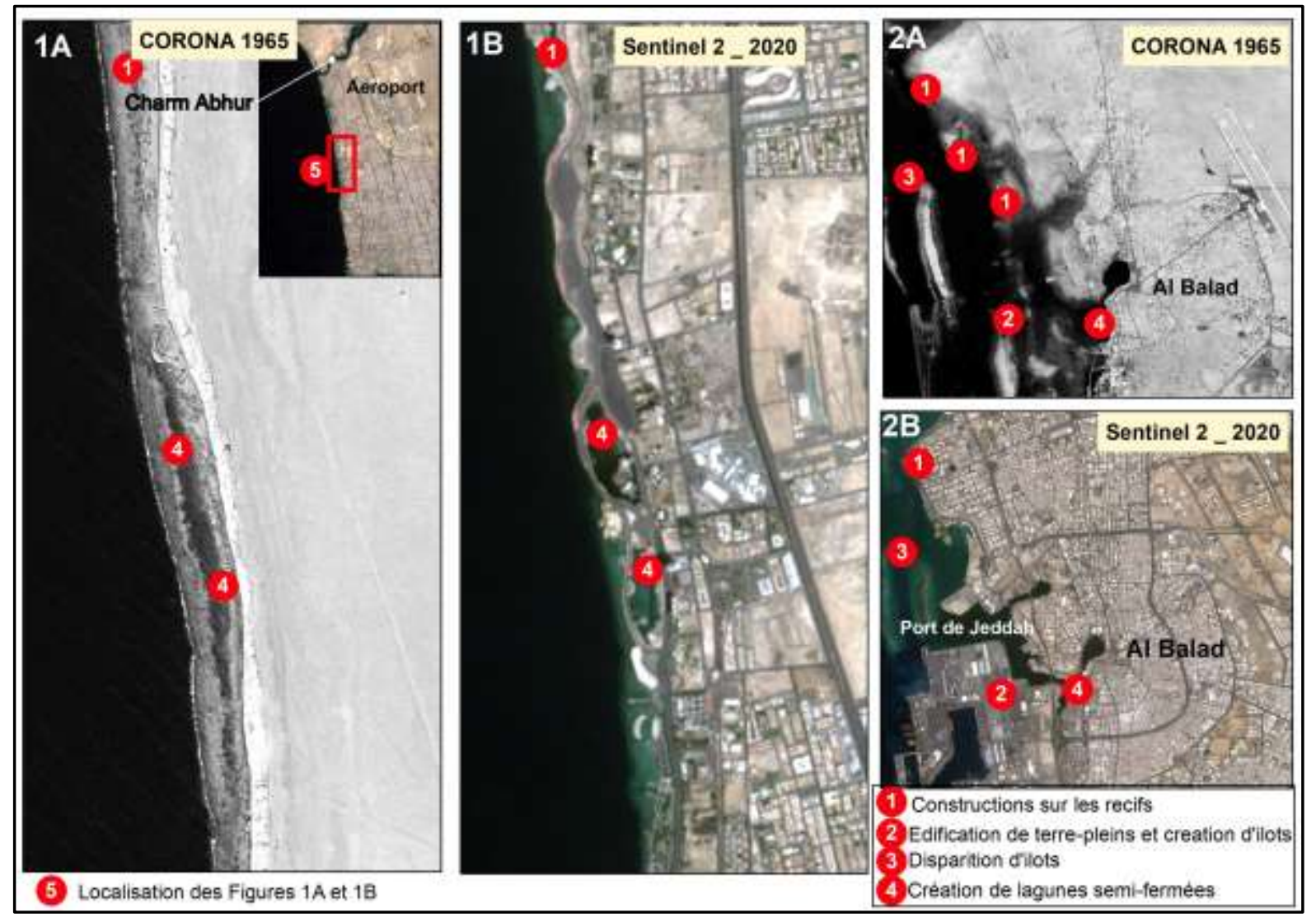

Figure 11. Illustration de différents changements dans la morphologie de la zone côtière et de la ligne de rivage au niveau de la zone du Port Islamique de Jeddah (2A et 2B) et au sud de Charm Abhur entre 1965 et 2020 .

\section{Les risques liés à la modification de la morphologie du littoral sont nombreux :}

L'extension des zones de basse altitude sur la frange littorale constitue une source majeure de risque d'inondation. Certains secteurs urbains de la ville de Jeddah sont construits sur l'emplacement d'anciennes lagunes ou de sebkhas. El Raey, (2009) a d'ailleurs identifié Jeddah parmi les zones littorales les plus vulnérables le long de la côte ouest de la mer Rouge.

- Les risques d'inondations sont par ailleurs exacerbés par des modifications au niveau des exutoires des oueds. Dans la zone étudiée l'extension de la plaine littorale modifie l'équilibre les oueds dont le parcours change pouvant augmenter les risques d'inondations.

- Les changements dans la topographie du littoral peuvent avoir une incidence sur toute la bande côtière : affaissement, constitution de sebkhas remontée des nappes salées. 
- La bathymétrie de la zone côtière et la géomorphologie littorale se retrouvent aussi complètement modifiées, la baie de Jeddah est en grande partie soit remblayée soit draguée,

\section{Discussion}

\section{Les principaux facteurs de la croissance urbaine (sur la mer) de Jeddah}

Il existe de nombreuses raisons qui ont conduit à l'extension urbaine sur la zone maritime et côtière. Les plus importantes sont la croissance démographique et urbaine ainsi que l'évolution de la fonction de la ville de Jeddah et de sa spécialisation.

\subsection{La fonction de la ville de Jeddah}

Les secteurs commercial et industriel sont devenus les principaux pourvoyeurs d'emplois au niveau de la ville de Jeddah à l'heure actuelle. Il convient de noter que les villes tirent leur fonction de la majorité du type d'activité de leur population, et à Jeddah, les travailleurs dans les activités secondaires et tertiaires atteignent 99\% de la maind'œuvre totale (L'autorité générale des statistiques, 2010), ce qui signifie que la majorité de la population travaille dans les activités administratives, de service, commerciales et industrielles. En conséquence, les activités de la population ont affecté le type d'utilisation des terres, notamment en ce qui concerne celles acquises au détriment de la mer

La fonction portuaire et administrative de Jeddah a été le principal facteur de croissance démographique de la ville, ce qui a entraîné une extension au détriment de la mer. La période où l'on note la plus grande part de terres gagnées sur la mer a été celle entre 1972 et 1986, Cette phase est marquée par l'émergence de plans de développement durable et d'ouverture commerciale à la suite des investissements dans le secteur pétrolier.

Le port de Jeddah et l'utilisation gouvernementale ont constitué le pourcentage le plus élevé dans les zones acquises au détriment de la mer tout au long de la période d'étude. L'utilisation touristique est apparue de manière intensive, en particulier au cours des dernières périodes en raison de l'adoption par le Royaume d'une politique économique, qui prône une diversification des ressources et la revitalisation du secteur du tourisme pour ne plus dépendre des seuls revenus pétroliers. 


\subsection{Extension de la ville de Jeddah}

La définition de la zone urbaine peut différer d'une source à l'autre selon la conception du bâti et non-bâti. Sont recensés comme faisant partie du bâti ou tissu urbain tous les terrains dans l'enceinte de la ville où un quelconque changement a été effectué par l'être humain, même si celui-ci consiste en un mur ou des fils pour délimiter un terrain, sans établir aucune installation dessus. À travers les différents images satellites, la zone urbaine de Jeddah a été délimitée et sa croissance est présentée au tableau 5 et aux figures 12 et 13.

Tableau 5. Etapes de la croissance urbaine à Jeddah entre 1965 et $2020^{*}$

\begin{tabular}{|c|c|c|c|c|}
\hline Année & $\begin{array}{c}\text { Superficie } \\
\mathbf{( k m}^{\mathbf{2}}\end{array}$ & $\begin{array}{c}\text { Accroissement } \\
\left.\mathbf{( k m}^{\mathbf{2}}\right)\end{array}$ & $\begin{array}{c}\text { \% de la superficie } \\
\text { actuelle }\end{array}$ & $\begin{array}{c}\text { Accroissement } \\
\text { moyen annuel }\end{array}$ \\
\hline 1965 & 35,1 & - & 3,1 & 0 \\
\hline 1972 & 74,8 & 39,7 & 6.6 & 5,6 \\
\hline 1986 & 592,5 & 517,7 & 52,7 & 36,9 \\
\hline 2003 & 728,2 & 135,7 & 64,8 & 7,9 \\
\hline 2020 & 1123,9 & 395,7 & 100 & 23,2 \\
\hline
\end{tabular}

*Certaines des nouvelles zones près de Jeddah, qui n'appartiennent pas aux activités urbaines ou à l'espace urbain, ont été négligées

La superficie totale de la ville de Jeddah était de $35,12 \mathrm{~km}^{2}$ en 1965 , ce qui signifie que la vieille ville ne représente que $3,1 \%$ de la superficie totale de la ville de à l'heure actuelle (figures 12 et 13).

La superficie de la ville a plus que doublé au cours des sept années suivantes, pour atteindre $74,8 \mathrm{~km}^{2}$ en 1972 , où elle représentait $6,6 \%$ de la superficie actuelle. Puis l'explosion urbaine a eu lieu et s'est reflétée sur l'image satellite de 1986, qui montre une zone urbaine atteignant $592,5 \mathrm{~km}^{2}$, soit $52,7 \%$ de la superficie actuelle. Cela signifie que la ville a augmenté chaque année pendant 14 ans d'une superficie plus grande que celle de la ville en 1965. Cette période (1972-1986) correspond au boom économique. Les investissements du gouvernement et du secteur privé bénéficient des recettes pétrolières par rapport au secteur public. Le secteur privé a également bénéficié de la facilitation gouvernementale et des prêts bancaires pour accroître la taille du secteur commercial et industriel de la ville. 


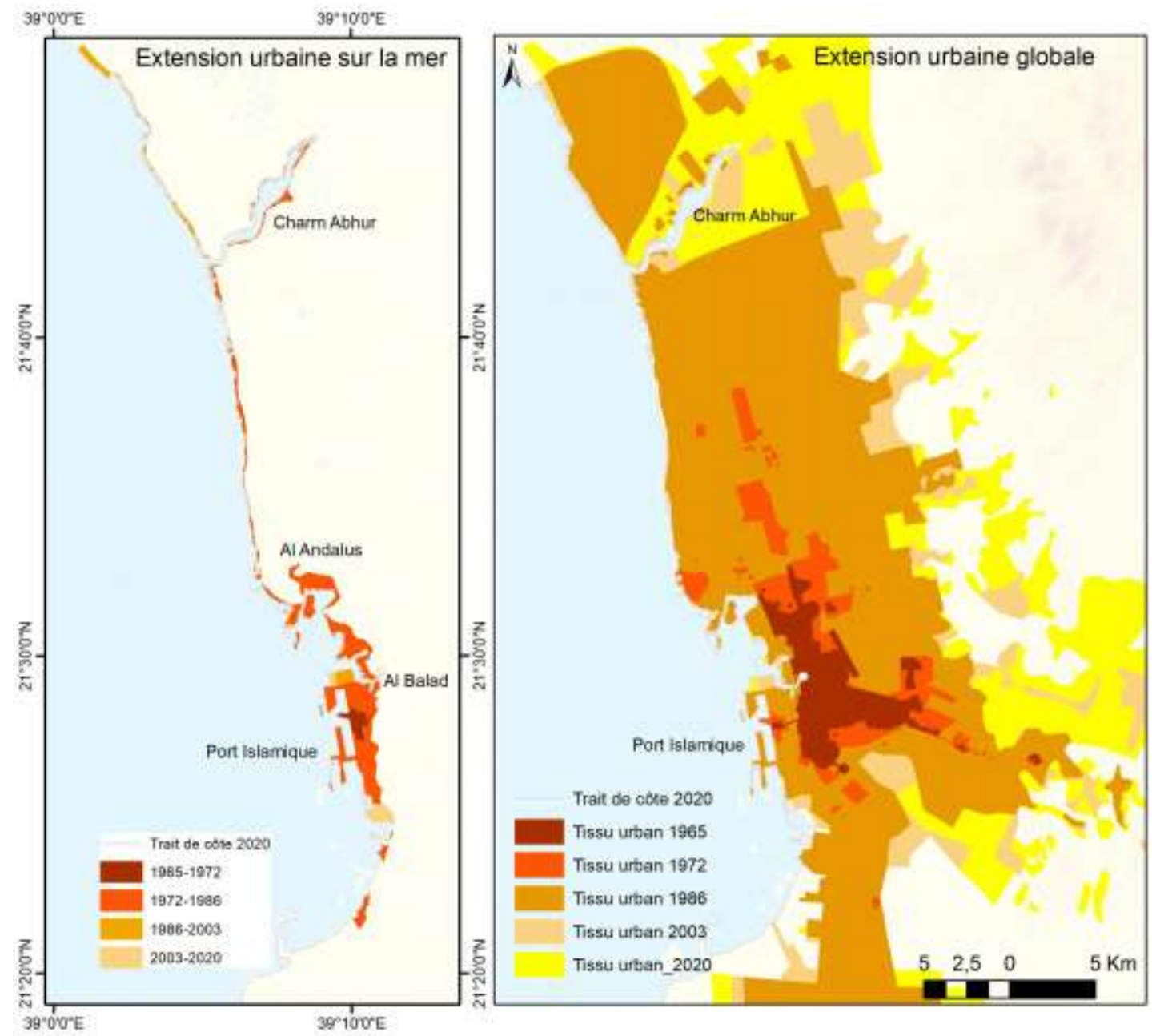

Figure 12. Les phases de l'extension urbaine de la ville (à droite) et au détriment de la mer (à gauche)

La croissance urbaine accélérée s'est arrêtée lors de période suivante jusqu'en 2003, et pendant 17 ans, la ville ne s'est étendue que de $135,7 \mathrm{~km}^{2}$, pour atteindre une superficie totale de $728,2 \mathrm{~km}^{2}$, soit une augmentation annuelle moyenne de seulement $7,9 \mathrm{~km}^{2}$. Ce ralentissement de l'expansion urbaine est peut-être dû à la forte augmentation urbaine de la période précédente ; des terres ont été acquises sans qu'il y ait des installations urbaines. Cette acquisition de terres était seulement destinée à l'appropriation foncière des terres au cours de cette période.

Actuellement, la ville s'est fortement développée pour atteindre en 2020 une superficie de 1123,9 km2, avec une augmentation moyenne annuelle de 23,2 km2 lors des 17 dernières années.

La figure 13 montre le taux de croissance, qui indique l'augmentation de la superficie de la ville entre deux dates successives. On remarque que la superficie a plus que doublé en 1972 que le taux le plus élevé est enregistré en 1986 où l'augmentation atteint $46 \%$ suivi par la dernière période $35 \%$. 


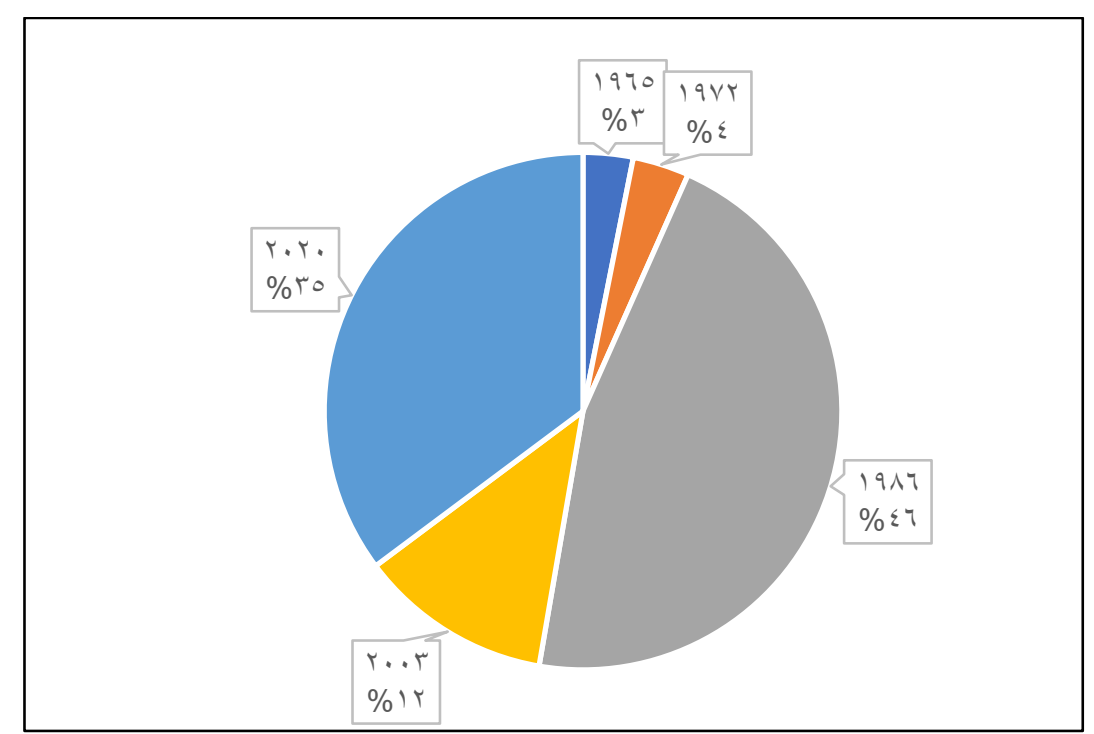

Figure 13. Taux de croissance urbaine Jeddah entre différentes dates

La majeure partie de cette augmentation s'est produite dans les directions du nord et de l'est et est exploitée à des fins résidentielles en fonction des plans de logement et des investissements immobiliers de l'État. Ces extensions sont également utilisées pour établir des centres de villégiature et des projets récréatifs dans le nord de Jeddah.

Il est évident que cette expansion urbaine lors des différentes périodes précédentes reflète clairement une forte demande urbaine qui pousse au remblaiement des zones côtières pour l'installation de divers types d'infrastructures et d'équipements publiques et privés pour des activités touristiques, récréatives, commerciales, résidentielles, gouvernementales, etc.

\subsection{Croissance démographique de la ville de Jeddah}

L'augmentation de la population est le principal moteur de la croissance urbaine de la ville, tant pour la disponibilisation des logements que des infrastructures, des services publics et sociaux et des activités économiques.

La ville a connu une croissance démographique plus rapide et plus importante que les autres agglomérations du royaume d'Arabie Saoudite. Jeddah était l'une des villes qui reflétait le mieux le développement économique du Royaume. Comme l'indiquent le tableau 6 et la figure 14, les périodes de plus forte croissance démographique se situent entre 1962 et 1974, où le taux de croissance démographique annuel a augmenté de $11 \%$. Ce taux est supérieur à celui du royaume, qui était de 7,4\%. Cette période marque le début du développement économique du Royaume du fait de 
la croissance économique dépendante des investissements pétroliers. Le taux de croissance élevé de la ville est aussi dû aux migrations que la ville a connues en raison de la disponibilité d'emplois, que ce soit dans la sphère gouvernementale ou privée.

Tableau 6. Population et taux de croissance démographique pour Jeddah et le royaume de 1962 à 2017

\begin{tabular}{|c|c|c|c|c|c|}
\hline \multirow[t]{2}{*}{ Année } & \multicolumn{2}{|c|}{ Population } & \multicolumn{2}{|c|}{ Taux de croissance annuel } & \multirow{2}{*}{$\begin{array}{l}\text { \% population Jeddah } \\
\text { par rapport au } \\
\text { royaume }\end{array}$} \\
\hline & Royaume & Jeddah & Royaume & Jeddah & \\
\hline 1962 & 3000000 & 147589 & & & 4.9 \\
\hline 1974 & 7009466 & 516104 & 7.3 & 11 & 7.4 \\
\hline 1992 & 16948388 & 2046251 & 5 & 8 & 12.1 \\
\hline 2004 & 22678262 & 2801481 & 2.5 & 2.7 & 12.4 \\
\hline 2010 & 27136977 & 3430697 & 3 & 3.4 & 12.6 \\
\hline 2017 & 32552336 & 4076000 & 2.6 & 2.5 & 13.4 \\
\hline
\end{tabular}

Source: General Authority for Statistics (KSA), (Mishkhes, 2000, p.81), Makkah Province, Jeddah Governorate, Annual Statistical Book of Saudi Arabia, 2017

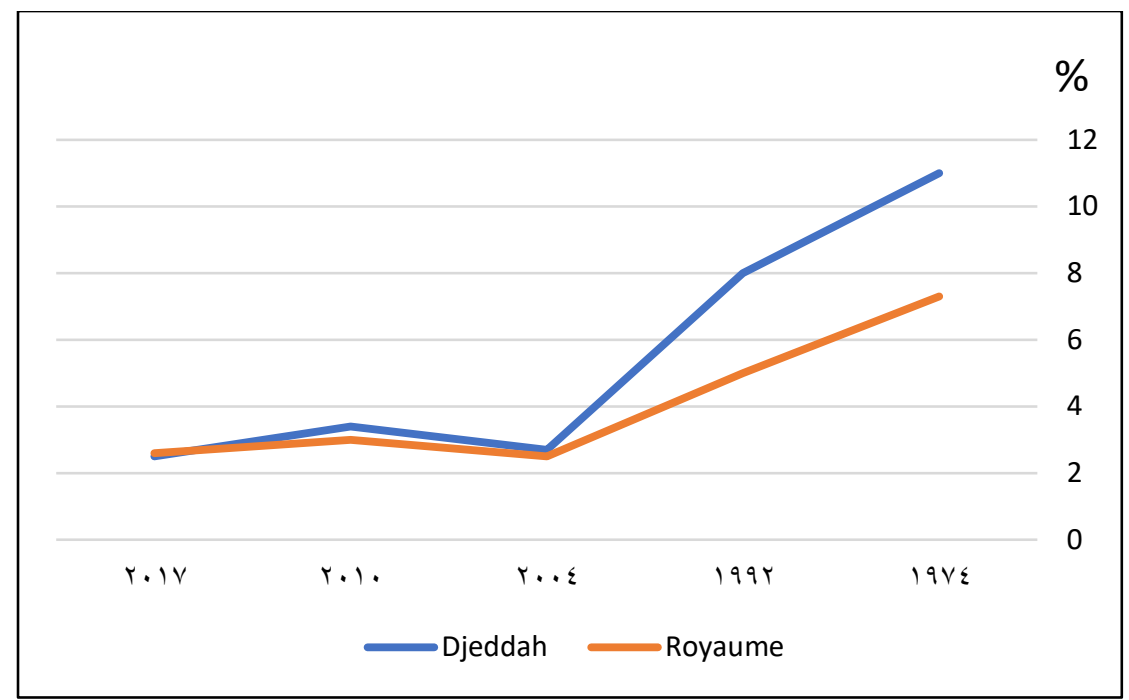

Figure 14. Les taux de croissance annuelles de la population à Djeddah et au Royaume au cours de la période de 1962 à 2017

Les migrations étaient souvent internes, des villages vers la ville, et donc l'augmentation est principalement liée aux Saoudiens qui viennent dans la ville pour chercher de l'emploi et de meilleurs services. Lors de la période suivante, de 1974 à 1992, la ville a également enregistré des taux de croissance démographique élevés, qui ont atteint $8 \%$ par an, alors que celui du Royaume était de $5 \%$. Cette période est considérée comme la fin de l'explosion démographique aux niveaux local et national. L'augmentation de la population au cours de cette période dépend des migrations internes ainsi que des migrations internationales, venant des pays en développement pour rechercher des emplois et améliorer leurs revenus (Abdulghani, 1983). Lors des 
périodes suivantes, les taux de croissance démographique de la ville et du royaume étaient stables, et la ville a maintenu les taux de croissance démographique les plus élevés du Royaume jusqu'à présent. En 56 ans, la ville de Jeddah concentre 13,4\% de la population du Royaume d'Arabie Saoudite, contre 4,9\% en 1962.

\section{Conclusion}

Cette recherche a permis de suivre l'extension de la ville de Jeddah à partir des données de télédétection multisources et multidates et de calculer les superficies urbaines gagnées sur la mer sur 55 ans entre 1965 et 2020. L'affectation de ces terres et les modifications morphologiques et environnementales engendrées ont également été analysées. Les SIG et les données de la télédétection ont été utilisés pour cartographier les surfaces artificialisées. Afin de mieux comprendre l'évolution spatiotemporelle des surfaces gagnées sur la mer et leur affectation, la durée de l'étude (55 ans) a été subdivisée en quatre périodes :1965-1972, 1972-1986, 1986-2003 et 2003-2020.

Les résultats obtenus indiquent que sur la période d'observation, la ville s'est étendue au détriment de la mer sur une superficie d'environ $33 \mathrm{~km}^{2}$ depuis 1965 . $\mathrm{Au}$ niveau des périodes l'annexion de zones maritimes au tissu urbain a atteint son paroxysme entre 1972 et 1986 avec une superficie de $24,5 \mathrm{~km}^{2}$ soit $75 \%$ de la surface totale des terre-pleins, ce qui s'explique par le boom économique (pétrolier) et démographique qui a prévalu à cette époque. La période 1965-1972 a enregistré la plus faible croissance urbaine au détriment de la mer, seule une superficie équivalente à 1,54 $\mathrm{km}^{2}$ a été édifiée. Ces remblaiements qui concernaient la partie centrale de la ville sont actuellement repartis le long de la côte. Le port de Jeddah a bénéficié de la plus grande zone d'expansion aux dépens de la mer, sa superficie a augmenté pendant toutes les périodes pour atteindre $11,8 \mathrm{~km}^{2}$ soit $36,1 \%$ de la superficie totale des terre-pleins. Ces différentes extensions lors des différentes phases représentent actuellement environ 94\% de la superficie totale de la zone industrialo-portuaire. En dehors du Port ces nouvelles terres sont affectées à divers usages ; gouvernemental/administratif (21,5\%), services publics $(16,4 \%)$, touristique /récréatif $(15,2 \%)$ et mixtes (résidentiel/commercial- 10,8\%).

Cette croissance urbaine dans ce secteur est soutenue par plusieurs facteurs dont (1) la fonction de la ville- marquée par prédominance des activités industrielles et 
commerciales ; (2) l'extension spatiale qui est passée d'environ $35 \mathrm{~km}^{2}$ en 1965 à 1123 $\mathrm{km}^{2}$ en 2020. La plus grande extension de la ville a également été relevée lors de la période entre 1972 et 1986 avec un accroissement d'environ $517 \mathrm{~km}^{2}$ (3) la croissance démographique plus rapide et plus forte que celles des autres villes du royaume.

Par ailleurs dans le sillage de l'extension urbaine dans les zones côtières, on assiste à des changements morphologiques et environnementales. La bathymétrie, la topographie de la zone côtière et la géomorphologie littorale sont modifiées suites aux draguages et aux remblaiements ; des ilots disparaissent ou apparaissent, des lagunes sont en partie fermées ou créées, des récifs coralliens sont détruits ou urbanisés, les traits de côtes sont modifiés. Une réduction de la superficie des mangroves et une destruction de l'environnement marin ont été également notées. 


\section{Références:}

Abdu M.S., Salagoor J.Y., An-Nwisser Al-Harigi F., 2002. Jeddah urban growth and development process: the underlying factors. Scientific Journal of King Faisal University (Basic and Applied Sciences) Vo.3 No.1 Dhu Al Hajjah 1422 (March 2002).

Abdulghani, K., 1983. Jeddah: A study of metropolitan change. Cities, Volume 10,

(1), feb 1993, Pages 50-59. https://doi.org/10.1016/0264-2751(93)90114-X

Aboulela, H.A., Bantan, R.A. \& Zeineldin, R.A. Evaluating and Predicting Changes Occurring on the Coastlines of Jeddah City Using Satellite Images. Arab J Sci Eng 45, 327-339 (2020). https://doi.org/10.1007/s13369-019-04085-1

Abu-Ouf M. and EI-Shater A., 1991. The relationship between the environmental conditions of the Jeddah coast, Red Sea, and benthic foraminifera. J. K.A. U. Mar. Sci., 2: 49-64.

Al Enezi, N.S A.A., 2019. Analysis of Urban Growth and Trends in Jeddah Using Remote Sensing Techniques and Geographic Information Systems. PhD Thesis, Umm Al Qura University, 269p.

Al Fara M. A. O. 1980, Géographie : étude analytique critique des concepts, écoles, tendances récentes de la recherche géographique. Publications département de geographie et association Koweitienne de Geographie, n 22 p14 (en arabe)

Aljaddani, A.H., 2015. Integration of multi-temporal remote sensing imagery and GIS for mapping and analysis of land use change in jeddah city, Saudi Arabia. Master Thesis, Murray, Kentucky USA.

Aljoufie M. (2020) Land Use, Transport, and Sustainability: Spatial Analysis of Commercial Centers and Public Transport Interaction in Jeddah. In: Bougdah H., Versaci A., Sotoca A., Trapani F., Migliore M., Clark N. (eds) Urban and Transit Planning. Advances in Science, Technology \& Innovation (IEREK Interdisciplinary Series for Sustainable Development). Springer, Cham

Almazroui, M., Mashat, A., Assiri, M.E. et al. Application of Landsat Data for Urban Growth Monitoring in Jeddah. Earth Syst Environ 1, 25 (2017). https://doi.org/10.1007/s41748-017-0028-4

Al-Sheikh A., 2011. Management of environmental degradation of Jeddah coastal zone, Saudi Arabia, using remote sensing and geographic information systems. Journal of American Science 2011;7(5):665-673.

Basaham, A.S. Rifaat, A.E., El-Sayed, M.A. and Rasul, N., 2006. Sharm Obhur: Environmental Consequences of 20 Years of Uncontrolled Coastal Urbanization. JKAU. Mar.Sci. Vol.17, pp129-152 (2006 A.D./1427 A.H.)

Cooper, J.A.G. et Alonso, I., 2006. Natural and anthropic coasts: challenges for coastal management in Spain. Journal of Coastal Research Special Issue 48. Coastal Geomorphology in Spain:

Daghistani, A.-M. I. (1993) 'A case study in planning implementation Jeddah, Saudi Arabia', Electronic working paper 5, University of Newcastle pp. 1-28. Available at: http://www.ncl.ac.uk/guru/assets/documents/ewp5.pdf.

Daoudi, M, and Niang, A, J. (April 3rd, 2019). Flood Risk and Vulnerability of Jeddah City, Saudi Arabia, Recent Advances in Flood Risk Management, John Abbot and Andrew Hammond, IntechOpen, DOI: 10.5772/intechopen.82073. 
EEA European Evironment Agency, 2006. « The changing faces of Europe's coastal areas » (report $n^{\circ}$ 6/2006). Luxembourg, Office des publications officielles des Communautés européennes. 107 p.

EL-Raey, 2009. Impact of Climate Change: Vulnerability and Adaptation Coastal Areas inTolba, M.K. and N. Saab (Edits: Arab Environment: Climate Change. Impact of climate Change on the Arab Countries; report of the arab forum for environment and development, afed, 2009.

EMCC (Economic and Management Consulting Center) 2017. Jeddah Facts and Figures. Chapter 3. Jeddah Annual Report 2016-2017.

Ewea H.A., 2010. Hydrological Analysis of Flooding Wastewater Lake in Jeddah, Saudi Arabia. JKAU: Met., Env. \& Arid Land Agric. Sci, 21, 125-144.

Helmi, M. R. (2015). The Ability of the Local Planning Authority to Implement Zoning Regulations: A Case Study of Jeddah, Saudi Arabia. PhD Thesis, Newcastle upon Tyne: Newcastle University.

Hooke, 1994. On the Efficacy of Humans as Geomorphic Agents. GSA today, 4 (9): 224-225. https://www.geosociety.org/gsatoday/archive/4/9/pdf/i1052-5173-4-9sci.pdf

Karim N., Wael E., Mahmod H., Fath A., Masria K.N. and Abdelazim N., 2018. Shoreline change detection using DSAS technique: Case of North Sinai coast, Egypt,Marine eoresources\&Geotechnology,10.1080/1064119X.2018.1448912.

L'autorité générale des statistiques, 2010. (General Authority for Statistics) Statistiques économiques 2010. https://www.stats.gov.sa/en/node

Le Berre et Robert, S.2017. L'urbanisation face à l'océan. Agathe Euzen; Françoise Gaill; Denis Lacroix; Philippe Cury. L'océan à découvert, CNRS éditions, pp.208-209, 2017, 978-2-271-11652-9. halshs-01626607

Le Berre, I., 2017. L'artificialisation des littoraux : déterminants et impacts. Béchet B.; Le Bissonais Y.; Ruas A. Sols Artificialisés et Processus d'artificialisation Des Sols : Déterminants, Impacts et Leviers d'action, Expertise Scientifique Collective (ESCo). IFSTTAR, INRA, pp.234-254, 2017. hal- 01764526

Luijendijk A., Roshanka G.H., GennadiiDonchyts F.B. and Aarninkhof S., 2018. The State of the World's Beaches. Scientific Reports, 2018; 8 (1) DOI: 10.1038/s41598-018-24630-6

Maki, M.C. 1995. Méthodes de recherche en géographie urbaine, King Abdulaziz University Journal of Educational sciences, 8 Recherches et articles p 169 (en arabe)

Mansour A.M., Madkour, H, A. 2015. Raised Coral Reefs and Sediments in the Coastal Area of the Red Sea in Rasul and I.C.F. Stewart (eds.), The Red Sea, Springer Earth System Sciences, DOI 10.1007/978-3-662-45201-1_23,

Martínez, M.L.; Intralawan, A.; Vázquez, G.; Pérez-Maqueo, O.; Sutton, P.; Landgrave, R., 2007. The coasts of our world: Ecological, economic and social importance. Ecological Economics, $63 \quad$ (2-3): 254-272. http://dx.doi.org/10.1016/j.ecolecon.2006.10.022

Mishkhes, M. 2000, Géographie humaine contemporaine du Royaume d'Arabie saoudite, deuxième édition, Dar Konooz Al-Maarefah, Djeddah (en arabe) 
Neumann, B.; Vafeidis, A.T.; Zimmermann, J.; Nicholls, R.J., 2015. Future Coastal Population Growth and Exposure to Sea-Level Rise and Coastal Flooding - A Global Assessment. Plos One, $10 \quad$ (3): 00118571. http://dx.doi.org/10.1371/journal.pone.0118571

Peter V. (2003). Jeddah's environmental problems. Geographical Review, Jul 2003; 93,3; ProQuest.

Sahahiri, R, Arrowsmith, C. \& Alitany, A.A. | (2019) Mapping the historical places: A case study of promoting tourism in Jeddah, the Kingdom of Saudi Arabia, Cogent Arts \& Humanities, 6:1, 1691315

Saudi Ports Authority 2019, Statistics of Year 2019

Spalding M., Green E., Ravilious, C., 2001. World Atlas of Coral Reefs. University of California Press, Berkeley.

Tezuka Akira, Takahashi Nobuo. La dynamique spatiale de la zone côtière autour de la baie de Tokyo. In : Hommes et Terres du Nord, 1998/4. Hommage à Pierre Bruyelle Aménagement du territoire et régions urbanisées. pp. 225-228; doi : https://doi.org/10.3406/htn.1998.2650

Tortel P. 2004. Thoughts on integrated coastal zone management (iczm) in saudiarabia The Regional Organization for the Conservation of the Environment of the Red Sea and Gulf of Aden. PERSGA report

Youssef A.M., Sefry S.A., Pradhan B. and Abu Alfadail E., 2016. Analysis on causes of flash flood in Jeddah city (Kingdom of Saudi Arabia) of 2009 and 2011 using multi-sensor remote sensing data and GIS, Geomatics, Natural Hazards and Risk, 7:3, 1018-1042, DOI: 10.1080/19475705.2015.1012750 